\title{
Pion and kaon parton distributions in the QCD instanton vacuum
}

\author{
Arthur Kock®, ${ }^{1, *}$ Yizhuang Liu, ${ }^{2, \dagger}$ and Ismail Zahed ${ }^{1, \$}$ \\ ${ }^{1}$ Department of Physics and Astronomy, Stony Brook University, Stony Brook, New York 11794-3800, USA \\ ${ }^{2}$ Tsung-Dao Lee Institute, Shanghai Jiao University, Shanghai 200240, China
}

(Received 10 April 2020; accepted 9 July 2020; published 23 July 2020)

\begin{abstract}
We discuss a general diagrammatic description of $n$-point functions in the QCD instanton vacuum that resums planar diagrams, and enforces gauge invariance and spontaneously broken chiral symmetry. We use these diagrammatic rules to derive the pion and kaon quasiparton amplitude and distribution functions at leading order in the instanton packing fraction for large but finite momentum. The instanton and antiinstanton zero modes and nonzero modes are found to contribute to the quasidistributions, but the latter are shown to drop out in the large-momentum limit. The pertinent pion and kaon parton distribution amplitudes and functions are made explicit at the low renormalization scale fixed by the inverse instanton size. Assuming that factorization holds, the pion parton distributions are evolved to higher renormalization scales with one-loop DGLAP evolution and compared to existing data.
\end{abstract}

DOI: 10.1103/PhysRevD.102.014039

\section{INTRODUCTION}

Light-cone distribution amplitudes are central to the description of hard exclusive processes with large momentum transfer. They account for the nonperturbative quark and gluon content of a hadron in the infinite-momentum frame. Using factorization, hard cross sections can be split into soft partonic distributions convoluted with perturbatively calculable processes. The soft partonic distributions are inherently nonperturbative. They can be extracted through moments using experiments [1], or more recently lattice simulations $[2,3]$.

Several QCD lattice simulations have suggested that the bulk characteristics and correlations of the light hadronic operators are mostly unaffected by lattice cooling [4], strongly suggesting that semiclassical gauge and fermionic fields may be dominant in the ground state. At weak coupling, instantons and anti-instantons are exact classical gauge tunneling configurations with large actions and finite topological charge which support exact quark zero modes with specific chirality.

Extensive analytical and numerical calculations [5-7], describing the QCD ground state as an ensemble of instantons and anti-instantons with hopping quark zero

\footnotetext{
*kock.arthur@stonybrook.edu yizhuang.liu@sju.edu.cn

*ismail.zahed@stonybrook.edu
}

Published by the American Physical Society under the terms of the Creative Commons Attribution 4.0 International license. Further distribution of this work must maintain attribution to the author(s) and the published article's title, journal citation, and DOI. Funded by SCOAP. modes were found to reproduce most of the cooled lattice simulations. In the quenched approximation, these calculations can be organized by observing that the ensemble is characterized by a small packing fraction in the large- $N_{c}$ limit which is dominated by planar graphs.

The twist-2 pion distribution amplitude and function have been discussed in the context of the QCD sum rules [8], bottom-up holographic models [9], bound-state resummations [10], basis light-front quantization using an effective Nambu-Jona-Lasinio (NJL) interaction [11], covariant NJL models with effective interactions [12], and the instanton model using nonlocal effective interactions and modified quark masses time-like [13,14].

The instanton model for the QCD vacuum is inherently space-like. It is amenable to QCD through semiclassics and allows for an organizational principle that enforces chiral and gauge Ward identities. It is well suited for the description of the bulk of the QCD vacuum with its flavor-singlet axial and scale anomalies, and its mesonic and baryonic excitations through pertinent Euclidean correlators [5-7]. However, its inherent Euclidean character makes it difficult to characterize the nonperturbative timelike structure of its partonic constituents as probed by deep inelastic scattering.

The recent suggestion put forth by $\mathrm{Ji}$ [2] and its matching protocol [15], to extract the light-cone distribution functions from equal-time quasidistributions in Euclidean space, has been carried on the lattice with some reasonable success [16]. This formulation makes the instanton calculus also ideal for a first-principle semiclassical analysis of the quasidistributions, and therefore the light-cone distributions by matching. Since the distribution functions obey rigorous sum rules, the enforcement of the Ward identities 
is important. This can be sought through a diagrammatic expansion and power counting around the planar approximation, both of which preserve chiral and gauge invariance. We note that recently, the quasipion distribution functions were analyzed using some of the models described above in Refs. [17,18].

The purpose of this paper is to revisit the $n$-point functions in the random instanton model in the planar approximation as in Ref. [19]. The latter resums a large class of instanton contributions in the form of nonperturbative integral equations with full chiral and gauge symmetry. While these equations are in general involved and require a numerical analysis, we will analyze them relying on the diluteness of the instantons and anti-instantons in the QCD vacuum, where the packing fraction is small with $\kappa \approx 10^{-3}$. All calculations will be carried at leading order (LO) and/or next-to-leading order (NLO) in $\alpha \approx \sqrt{\kappa}$.

This expansion provides an organizational principle and addresses some of the shortcomings in Refs. [6,7] by enforcing the axial and vector Ward identities in the planar approximation. It turns out that the zero modes and nonzero modes are equally important in this enforcement, as initially noted for the two-point vector correlator in Ref. [20]. Also, the virtual character of the induced effective quark constituents generated by the planar resummation makes their time-like manifestation in the pion distribution amplitude and function very subtle.

This paper consists of several new results: 1) a generalization of the planar resummation to the $n$-point functions; 2) an explicit derivation of the two- and three-point functions at NLO; 3) a derivation of the quark effective mass at NLO; 4) a planar resummation of the pion quasiparton amplitude and distribution functions at LO; 5) explicit expressions for the pion and kaon parton distribution amplitude (PDA), parton distribution function (PDF) and transverse momentum distribution (TMD) at LO; 6) an explicit proof of the axial Ward identity for the axial-axial correlation function at NLO; and 7) the explicit evolution of the pion PDA and PDF and a comparison with currently available data.

The organization of the paper is as follows. In Sec. II we briefly review the general aspects of the random instanton vacuum, and detail the planar approximation for the derivation of the quark propagator. We derive the induced effective quark mass and in general the spin-valued selfenergy at NLO. In Sec. III we show how the two-point meson correlators are resummed, and use the result to derive the pion pseudoscalar vertex at NLO. The pion decay constant is worked out in the leading-logarithm approximation. In Sec. IV we derive the pion quasiparton distribution amplitude (QPDA) at LO in the leadinglogarithm approximation and beyond. In Sec. V we show how the planar resummation applies to the three-point functions and use it to construct the pion valence quasidistribution function (QPDF), PDF and TMD also at LO. Our conclusions are in Sec. VI. A number of details can be found in several appendixes, including the LO result for the pion generalized distribution function (GPDF).

\section{QUARK PROPAGATOR}

Key to the analysis of the spontaneous breaking of chiral symmetry in the random instanton vacuum is the dynamic appearance of a momentum-dependent constituent mass. To illustrate this, consider the quark propagator in the chiral limit and the quenched approximation of QCD

$$
\left\langle\psi(x) \psi^{\dagger}(y)\right\rangle=\left\langle\left\langle x\left|(-i \not \partial-\not A-i m)^{-1}\right| y\right\rangle\right\rangle_{A}
$$

where the averaging is over the gauge configurations $A$. In the instanton vacuum, the gauge configurations are restricted to the semiclassical set of instantons and antiinstantons which are sampled either randomly (random instanton model) or through their semiclassical interactions (interacting instanton model). Throughout, we will refer to the instanton liquid model as the random instanton model. With this in mind and to enforce topological neutrality of the vacuum, the semiclassical configurations are chosen to be $\frac{N}{2}$ instantons and $\frac{N}{2}$ anti-instantons noninteracting and randomly distributed in a 4-volume $V_{4}$, with

$$
A=\sum_{I=1}^{\frac{N}{2}} A_{I}+\sum_{\bar{I}=1}^{\frac{N}{2}} A_{\bar{I}} .
$$

The instanton and anti-instanton $A_{I, \bar{I}}$ in Eq. (2) are each represented by an $\mathrm{SU}(2)$-valued matrix embedded in $\mathrm{SU}\left(N_{c}\right)$ with arbitrary orientation $U_{I, \bar{I}}$ in color space and position $z_{I, \bar{I}}$ in 4-space. For simplicity, all instantons are chosen to have the same size $\rho \approx 1 / 3 \mathrm{fm}$ with a density fixed at $n=N / V_{4} \approx 2 \mathrm{fm}^{-4}$. In Fig. 1 we show the instanton size distribution versus their size $\rho$ in fm from the instanton liquid model in comparison to $\mathrm{SU}(2)$ lattice simulations [5,21].

In the semiclassical background (2) the quark propagator can be organized as an expansion in multiple rescatterings with increasing numbers of instantons and anti-instantons

$$
\begin{aligned}
S-S_{0}= & \left\langle\sum_{I} S_{I}-S_{0}+\sum_{I \neq J}\left(S_{I}-S_{0}\right) S_{0}^{-1}\left(S_{J}-S_{0}\right)\right. \\
& \left.+\sum_{I \neq J, J \neq K}\left(S_{I}-S_{0}\right) S_{0}^{-1}\left(S_{J}-S_{0}\right) S_{0}^{-1}\left(S_{K}-S_{0}\right)+\cdots\right\rangle .
\end{aligned}
$$

Here $S_{0}=1 /(-i \not \partial-i m)$ is the free quark propagator, $S_{I}=1 /\left(-i \not \supset-A_{I}-i m\right)$ is the quark propagator in a single instanton or anti-instanton background and the sum is over all instantons and anti-instantons. The averaging denotes independent integrations over the instanton and anti-instanton positions and color orientations. 


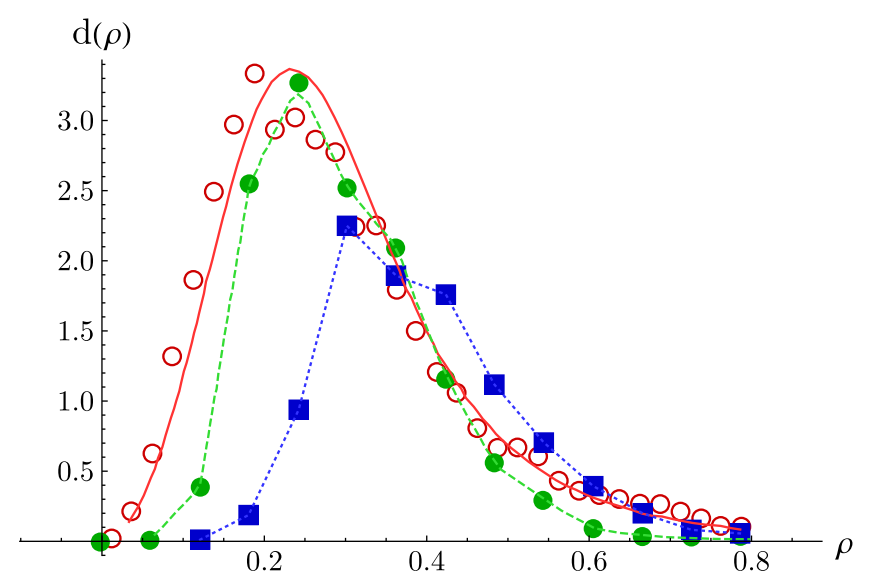

FIG. 1. Instanton size distribution in pure SU(2) gauge theory calculated on the lattice [21] and in the interacting instanton liquid model (IILM) [5]. The unit scale is fm. Closed points correspond to lattice data with decreasing lattice spacing. Open points are from an IILM calculation. The solid line is a parametrization of the IILM result as discussed in Ref. [5]. The dotted and dashed lines simply aid the eye. In both pure SU(2) and pure $\mathrm{SU}(3)$, an average value $\rho \approx 1 / 3 \mathrm{fm}$ is found.

\section{A. Planar resummation}

Large- $N_{c}$ QCD is essentially a quenched approximation dominated by planar graphs. The same applies to its semiclassical approximation in terms of a random instanton vacuum, if we note that $n \sim N_{c}$ and that the averaging over the $\mathrm{SU}\left(N_{c}\right)$ color orientations provides extra factors of $1 / N_{c}$. The planar contributions to the quark propagator are resummed through the formal equation [19]

$$
\begin{aligned}
S-S_{0} & =\frac{N}{2 V_{4}} \int_{I+\bar{I}} d z_{I} d U_{I}\left(\frac{1}{1-S_{Y} \Delta}\right) S_{Y} S_{0}^{-1} S \\
& =\frac{N}{2 V_{4}} \int_{I+\bar{I}} d z_{I} d U_{I} S_{0}\left(\frac{1}{S_{0} S_{Y}^{-1} S_{0}-S_{0} \Delta S_{0}}\right) S
\end{aligned}
$$

with the single instanton (anti-instanton) propagator $S_{I}=S_{0}+S_{Y}$, and the amputated and resummed propagator

$$
S_{0}^{-1} S S_{0}^{-1}=\Delta+S_{0}^{-1} .
$$

The integrations in Eq. (4) are over the instanton moduli for fixed instanton size. The integration over the rigid gauge color $U_{I}$ projects onto the color-singlet channel, while the integration over the global 4-position $z_{I}$ restores translational invariance. Equation (4) is readily recast in the form

$$
S^{-1}-S_{0}^{-1}=\frac{N}{2 V_{4}} \int_{I+\bar{I}} d z_{I} d U_{I}\left(\frac{1}{S-S_{0}\left(S_{0}^{-1}+S_{Y}^{-1}\right) S_{0}}\right) .
$$

Inserting the identity

$$
S_{Y}^{-1}=\frac{1}{S_{I}-S_{0}}=S_{0}^{-1}\left(-A_{I}\right)^{-1} S_{I}^{-1}
$$

in Eq. (6) yields a formal integral equation for the selfenergy [19]

$$
\begin{aligned}
S^{-1}-S_{0}^{-1} & =\frac{N}{2 V_{4}} \int_{I+\bar{I}} d z_{I} d U_{I} \frac{1}{S-A_{I}^{-1}} \\
& \equiv \frac{N}{2 V_{4}} \int_{I+\bar{I}} d z_{I} d U_{I} \Sigma_{I}
\end{aligned}
$$

which sums over a single instanton plus anti-instanton. The $\mathrm{SU}\left(N_{c}\right)$ averaging in Eq. (8) over $U_{I}$ projects onto the color-singlet channel, and the $z$ integral restores translational invariance. Throughout and for simplicity, only the massless case will be considered with $m=0$ unless specified otherwise.

For $S^{-1}-S_{0}^{-1}=-i \sigma$, Eq. (8) takes the formal gap-like form

$$
i \sigma=\frac{N}{2 N_{c} V_{4}} \int_{I+\bar{I}} d z_{I} \operatorname{Tr}_{C}\left(A_{I} \frac{1}{i \not \partial+\not A_{I}+i \sigma}(i \not \partial+i \sigma)\right)
$$

with $\operatorname{Tr}_{C}$ referring to the color trace (in some places below it will also mean spin as well). The small parameter $\kappa$ or packing fraction,

$$
\kappa=\frac{N \rho^{4}}{2 V_{4} N_{c}} \equiv \alpha^{2} \rho^{4} \approx 3.186 \times 10^{-3}
$$

is of order $N_{c}^{0}$ provided that the instanton density is made to scale as $N / V_{4} \sim N_{c}$.

\section{B. Effective mass at LO}

The effective mass operator (8) can be sought iteratively $\sigma=\alpha \sigma_{0}+\alpha^{2} \sigma_{1}+\cdots$, with a starting correction of order $\alpha$ and not $\alpha^{2}$ [19]. At LO,

$\Delta_{I}(x, y) \equiv\left\langle x\left|\frac{1}{i \not \partial+A_{I}+i \alpha \sigma_{0}}\right| y\right\rangle=\frac{\psi_{0 I}(x) \psi_{0 I}^{\dagger}(y)}{i \alpha \sigma_{00}}+\mathcal{O}(\alpha)$

where $\sigma_{00}=\left\langle 0\left|\sigma_{0}\right| 0\right\rangle=\sqrt{2}\left\|q \varphi^{2}\right\| \sim \rho$ is the expectation value of $\sigma_{0}$ in the zero-mode state $\psi_{0 I}$. Its form is given in Appendix A both in regular and singular gauge. The norm notation is subsumed. Inserting Eq. (11) into Eq. (9) yields the effective mass at LO 


$$
M(p) \approx \alpha \sigma_{0}(p)=\frac{\alpha}{\sigma_{00}} \operatorname{Tr}_{C}\left(\not p\left(\psi_{0 I}(p) \psi_{0 I}^{\dagger}(p)+\psi_{0 \bar{I}}(p) \psi_{0 \bar{I}}^{\dagger}(p)\right) \not \supset\right) \equiv \frac{\alpha}{\sigma_{00}} \operatorname{Tr}_{C}\left(\not p\left(P_{I}(p)+P_{\bar{I}}(p)\right) \not p\right)=\frac{\alpha}{\sqrt{2}} \frac{\left|p \varphi^{\prime}(p)\right|^{2}}{\left\|q \varphi^{\prime 2}\right\|}
$$

where $P_{I}$ is the zero-mode projector. After color tracing, the effective mass operator is diagonal in spin. The last relation holds for both the regular and singular gauge, but with different zero-mode profiles. In singular gauge from Appendix A we have

$$
\frac{M(p)}{M(0)}=\left(\left|z\left(I_{0} K_{0}-I_{1} K_{1}\right)^{\prime}\right|^{2}\right)_{z=\frac{1}{2} \rho p}
$$

The effective mass is not analytic in the packing fraction since $\alpha \sim \sqrt{\kappa}$. In Fig. 2 we show Eq. (12) for $M(0)=383 \mathrm{MeV}$ and $\rho=0.313 \mathrm{fm}$ (solid red curve) in comparison to the lattice-generated effective quark mass in Coulomb gauge [22]. The spread corresponds to a 99\% confidence interval generated by a standard weighted least-squares regression on the data in Fig. 2, giving the parameter ranges $M(0)=383 \pm 39 \mathrm{MeV}$ and $\rho=$ $0.313 \pm 0.016 \mathrm{fm}$. At large momenta, $M(p) \approx 1 / p^{6}$ falls faster than $\alpha_{s}^{D}\left(p^{2}\right) / p^{2}$ with $D=39 / 27$ derived using short-distance QCD arguments [23], but in good agreement with the lattice results.

\section{Effective mass at NLO}

At NLO the effective mass is obtained by further expanding Eq. (11) and keeping the contributions to order $\alpha$. For that, we can organize Eq. (11) formally so that

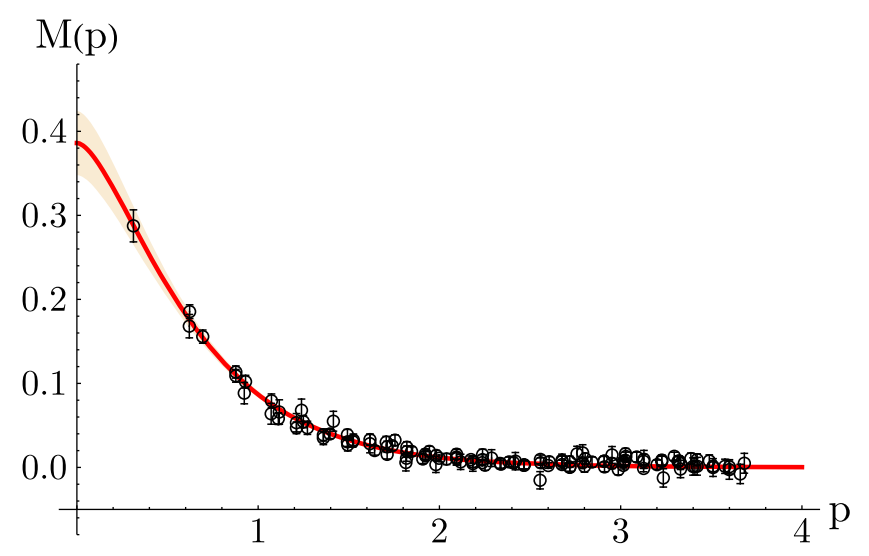

FIG. 2. Momentum dependence of the instanton-induced effective quark mass in singular gauge (13) at LO (solid curves), compared to the effective quark mass measured on the lattice in Coulomb gauge [22] (open circles). The unit scale is $\mathrm{GeV}$. We obtain the fitted parameter intervals $M(0)=383 \pm$ $39 \mathrm{MeV}$ and $\rho=0.313 \pm 0.016 \mathrm{fm}$.

$$
\begin{aligned}
\Delta_{I} & =\frac{1}{i \not \partial+\mathscr{A}_{I}+i \alpha \sigma_{00}}+\frac{1}{i \not \partial+\mathscr{A}_{I}+i \alpha \sigma_{00}}\left(-i \alpha\left(\sigma_{0}-\sigma_{00}\right)\right) \Delta_{I} \\
& =\left(\frac{P_{I}}{i \alpha \sigma_{00}}+G_{I}\right)+\left(\frac{P_{I}}{i \alpha \sigma_{00}}+G_{I}\right)\left(-i \alpha\left(\sigma_{0}-\sigma_{00}\right)\right) \Delta_{I}
\end{aligned}
$$

where $P_{I}$ is the projector on the quark zero mode, and $G_{I}$ is the quark nonzero-mode propagator in the instanton background. Using the virtual quark eigenstates $\left(i \not \partial+\mathscr{A}_{I}\right) \psi_{k I}=$ $E_{k} \psi_{k I}$ and the mode expansion for $G_{I}=\sum_{k \neq 0} \psi_{k I} \psi_{k I}^{\dagger} / E_{k}$ we can explicitly solve for $\Delta_{I}$ at NLO

$$
\begin{aligned}
\Delta_{I}= & \frac{P_{I}}{i \alpha \sigma_{00}}+\sum_{k \neq 0} \frac{\psi_{k I} \psi_{k I}^{\dagger}}{E_{k}}+P_{I} \sum_{k \neq 0} \frac{\sigma_{0 k} \sigma_{k 0}}{E_{k} \sigma_{00}} \\
& -\sum_{k \neq 0} \frac{\psi_{k I} \psi_{0 I}^{\dagger} \sigma_{k 0}}{E_{k} \sigma_{00}}-\sum_{k \neq 0} \frac{\psi_{0 I} \psi_{k I}^{\dagger} \sigma_{0 k}}{E_{k} \sigma_{00}}+\mathcal{O}(\alpha) .
\end{aligned}
$$

The matrix elements $\sigma_{k l}=\langle k|\sigma| l\rangle$ involve $\sigma$ only at leading order or $\sigma_{0}$. From Eq. (9) the self-energy at NLO is spin valued and reads

$$
\begin{aligned}
\Sigma_{I}= & A_{I} \frac{-1}{i \not \partial+\not A_{I}+i \sigma}(i \not \partial+i \sigma) \\
\approx & i \not \partial \frac{P^{I}}{i \alpha \sigma_{00}} i \not \partial+i \not \partial\left(1-P^{I} \hat{\sigma}\right) G^{I}\left(1-\hat{\sigma} P^{I}\right) i \not \partial-i \not \supset \\
& +i \not \partial P^{I} \hat{\sigma}+\hat{\sigma} P^{I} i \not \partial-\frac{\beta_{00}}{i \sigma_{00}^{2}} i \not \partial P^{I} i \not \partial \approx \frac{\Sigma_{I 0}}{\alpha}+\Sigma_{I 1}
\end{aligned}
$$

with $\hat{\sigma}=\sigma_{0} / \sigma_{00}$. The last contribution was added to account for the planar resummation to the self-energy through the gap equation

$$
-i \beta_{00}(p)=\int d^{4} x d^{4} y e^{i p \cdot(x-y)} \operatorname{Tr}_{C} \Sigma_{I 1}(x, y)=\operatorname{Tr}_{C} \Sigma_{I 1}(p, p) \text {. }
$$

The anti-instanton contribution follows from the substitution $I \rightarrow \bar{I}$. The effective mass at NLO follows by substituting Eq. (16) into Eq. (9) which is now a true gap equation because of the contribution (17). The first contribution is in agreement with Eq. (12). The nonzero-mode contribution $G_{I}$ in Eq. (16) is important for the enforcement of symmetries at NLO. Its explicit form in singular gauge is given in Appendix A.

\section{MESONIC VERTICES IN THE PLANAR APPROXIMATION}

In this section we formulate the planar resummation for the two-point functions. We will focus on flavor-singlet 
correlators with a single flavor and ignore vacuum loops, with the assumption that we are evaluating nonsinglet flavor correlators where the loop corrections are suppressed by $1 / N_{c}$. We will analyze in detail the resummation for the two-point pseudoscalar source at LO and NLO.

\section{A. Meson correlators}

The $T$ matrix in the planar approximation for rescattering of two quarks in the $2 \rightarrow 2$ channel, can be formally constructed using the two-particle irreducible (2PI) kernel

$$
K=\frac{N}{2 V} \int_{I+\bar{I}} d z_{I} d U_{I}\left(\Sigma_{I} \otimes \Sigma_{I}\right)
$$

with the color-spin-space-valued self-energy $\Sigma_{I}$ given in Eq. (8). We can now use the 2PI kernel to construct any two-point correlation function in the QCD instanton vacuum. For that, consider the general local and colorless source $J_{\Gamma}=\psi^{\dagger} \Gamma \psi$, say for a meson of spin flavor $\Gamma$, and define the amputated spin-flavor-valued operator

$$
O_{\Gamma a b}(P, k)=S^{-1}(k)\left\langle\psi_{a}(k) \psi_{b}^{\dagger}(P-k) J_{\Gamma}(P)\right\rangle S^{-1}(k-P)
$$

where the averaging is carried over the instanton-antiinstanton vacuum gauge configurations. Equation (19) refers to a quark of color-spin $a$ and momentum $k$ combining with a quark of color-spin $b$ and momentum $P-k$ to give a colorless meson of spin $\Gamma$ and momentum $P$.

The resummed instanton contributions to Eq. (19) in the planar approximation can be obtained by tracing Eq. (19) with the 2PI kernel (18) with the result

$$
\begin{aligned}
O_{\Gamma}(P, k)= & \Gamma+\alpha^{2} \rho^{4} \sum_{I+\bar{I}} \int \frac{d^{4} p}{(2 \pi)^{4}} \operatorname{Tr}_{C}\left(\Sigma_{I}(k, p)\right. \\
& \left.\times S(p) O_{\Gamma}(P, p) S(p-P) \Sigma_{I}(p-P, k-P)\right) .
\end{aligned}
$$

The net effect of averaging over the instanton-antiinstanton ensemble is the projection onto the color-singlet channel and momentum conservation. Equation (20) is the analogue of the Bethe-Salpeter equation for the vertex functions. Using Eq. (20) the resummed two-point function for an arbitrary meson correlator in the planar approximation is

$$
\begin{aligned}
\mathcal{C}_{\Gamma_{1} \Gamma_{2}}(P) & \equiv\left\langle O_{\Gamma_{1}}(-P) O_{\Gamma_{2}}(P)\right\rangle \\
& =-\int \frac{d^{4} k}{(2 \pi)^{4}} \operatorname{Tr}_{C}\left(\Gamma_{1} S(k) O_{\Gamma_{2}}(P, k) S(k-P)\right) .
\end{aligned}
$$

\section{B. Pseudoscalar pion vertex at LO}

The leading-order $\alpha$ contribution to the pion pseudoscalar correlator can be obtained by setting $\Gamma=\gamma_{5}, \Sigma_{I} \sim \Sigma_{0} / \alpha$ and $S \sim 1 /\left(\not K-i \alpha \sigma_{0}(0)\right)$ in Eqs. (20)-(21). As will be seen, many of our expressions will in fact be (logarithmically) divergent in $\alpha$. We will encounter integrals of the form

$$
\int \frac{d^{4} k}{(2 \pi)^{4}} \frac{F(k)}{\left(k^{2}+\alpha^{2} \sigma_{0}^{2}(0)\right)}
$$

where $F(k)$ is a smooth function that approaches a constant value as $k^{2} \rightarrow 0$ and rapidly drops off as $k^{2} \gg \rho^{-2}$. To extract the leading divergent behavior from an integral of this kind, we may shift $k^{2} \rightarrow k^{2}-\alpha^{2} \sigma_{0}^{2}(0)$, and subsequently drop corrections to $F\left(k^{2}-\alpha^{2} \sigma_{0}^{2}(0)\right) \approx F\left(k^{2}\right)$ which only contribute subleading divergent corrections [e.g., $\alpha \log (\alpha), \alpha^{2} \log (\alpha)$, etc.]. As will prove useful later, it is totally equivalent to instead only shift $k_{\perp}^{2} \rightarrow$ $k_{\perp}^{2}-\alpha^{2} \sigma_{0}^{2}(0)$, which then leads to a shift in the lower bound of integration of only $k_{\perp}^{2}$,

$$
k_{\perp}^{2}>\alpha^{2} \sigma_{0}^{2}(0)=M^{2}(0) .
$$

With this in mind, a formal solution follows for the vertex operator $O_{5}(P, k) \approx \gamma^{5} F_{5}(P, k)$, where the vertex function $F_{5}(P, k)$ is diagonal in spin space. In singular gauge, the latter formally satisfies the integral equation

$$
\gamma^{5} F_{5}(P, k)=\gamma^{5}+\frac{\varphi^{\prime}(k) \varphi^{\prime}(k-P)|k||k-P|}{\sigma_{00}^{2}} \int \frac{d^{4} p}{(2 \pi)^{4}} F_{5}(P, p)\left(-\psi_{I}^{\dagger}(p) \psi_{I}(p-P) \frac{1-\gamma_{5}}{2}+\psi_{\bar{I}}^{\dagger}(p) \psi_{\bar{I}}(p-P) \frac{1+\gamma_{5}}{2}\right) .
$$

This is a Fredholm integral equation of the second kind, and can be solved with a Liouville-Neumann series, which is found to be geometric. The solution then follows by summation with the result

$$
F_{5}(P, k)=1+\lambda(P) \frac{\varphi^{\prime}(k) \varphi^{\prime}(k-P)|k||k-P|}{\sigma_{00}^{2}}
$$

with $\lambda(P)$ satisfying

$$
\lambda(P)\left(1-\frac{2}{\sigma_{00}^{2}} \int \frac{d^{4} k}{(2 \pi)^{4}} \varphi^{\prime 2}(k) \varphi^{2}(k-P)\left(k^{2}-k \cdot P\right)\right)=1
$$


For small momentum we have $\lambda(P) \approx\left(\sqrt{N_{c}} / f_{\pi}\right) / P^{2}$, corresponding to the pion pole in the chiral limit. We note that the LO contribution (25) amounts to the effective coupling at the pion pole

$\left(i O_{5}(P, k)\right)_{P^{2} \approx 0} \approx \frac{\sqrt{N_{c}}}{f_{\pi}} \sqrt{M(k)}\left(\frac{i \gamma_{5}}{P^{2}}\right) \sqrt{M(k-P)}$

with the running mass of order $\alpha$ given in Eq. (12). This LO result is in agreement with the effective vertex following from the partially resummed planar diagrams in Refs. [5-7]. Equation (25) gives the full pion pseudoscalar vertex on and off mass shell in the massless limit. The massive case will be discussed below.

\section{Pion decay constant $f_{\pi}$ at LO}

The explicit values of $g_{\pi}$ and $f_{\pi}$ follow from the pseudoscalar two-point correlation function (21) with the vertex function (25), which is of order $\alpha^{0}$ to LO. More specifically, the pion-pole contribution reads

$\mathcal{C}_{\gamma_{5} ; \gamma_{5}}(P) \approx-\int \frac{d^{4} k}{(2 \pi)^{4}} \operatorname{Tr}\left(\gamma_{5} F_{5}(P, k) \frac{1}{\not k} \gamma_{5} \frac{1}{\not k-\not P}\right) \approx \frac{g_{\pi}^{2}}{P^{2}}$.

$g_{\pi}$ defines the pseudoscalar pion-quark-quark coupling and at $\mathrm{LO}$ is given by

$$
\begin{aligned}
g_{\pi}^{2}= & -N_{c}\left(\int \frac { d ^ { 4 } k } { ( 2 \pi ) ^ { 4 } } \left(3 \varphi^{4}+7(k \rho) \varphi^{\prime 3} \varphi^{\prime \prime}\right.\right. \\
& \left.\left.+(k \rho)^{2}\left(\varphi^{\prime 3} \varphi^{\prime \prime \prime}+\varphi^{\prime 2} \varphi^{\prime 2}\right)\right)\right)^{-1}
\end{aligned}
$$

The pion decay constant $f_{\pi}$ is related to $g_{\pi}$ in Eq. (29) by chiral reduction with

$$
f_{\pi} g_{\pi}=-2 i\left\langle\psi^{\dagger} \psi\right\rangle \approx 2 N_{c} \frac{M(0)}{(2 \pi \rho)^{2}}
$$

at leading order in the current quark mass. The last identity is the LO contribution to the chiral condensate, and is the expected Goldberger-Treiman relation for the effective quark coupling. Equation (30) is infrared finite and of order $\alpha$. Both $f_{\pi}$ and $g_{\pi}$ are infrared sensitive with

$$
g_{\pi}^{2} \approx N_{c}\left(\int \frac{d^{4} k}{(2 \pi)^{4}} \frac{(2 \pi \rho)^{4}}{k^{4}}\right)^{-1}
$$

and similarly for $f_{\pi}$

$$
f_{\pi}^{2} \approx 4 N_{c} \int \frac{d^{4} k}{(2 \pi)^{4}} \frac{M^{2}(0)}{k^{4}} .
$$

Note that $g_{\pi}$ in Eq. (29) and therefore $f_{\pi}$ in Eq. (30) are UV finite with an approximate range of $1 / \rho$. The infrared sensitivity follows from the shift which led to Eq. (23). This shift will be understood throughout.

To logarithmic accuracy $g_{\pi}^{2} \sim N_{c} / \ln (1 / \alpha)$ and $f_{\pi}^{2} \sim$ $N_{c} \alpha^{2} \ln (1 / \alpha)$ with

$$
f_{\pi}^{2} \approx \frac{N_{c} M^{2}(0)}{2 \pi^{2}} \ln \left(\frac{C}{\rho M(0)}\right)
$$

modulo a constant $C$ of order $1 . f_{\pi}^{2}$ captures the chiral conductivity in the QCD vacuum [24]. Using the values of $M(0) \rho$ displayed in Fig. 2 we have $C=0.849$ for $f_{\pi}=$ $86 \mathrm{MeV}$ in the chiral limit, $C=0.897$ for $f_{\pi}=93 \mathrm{MeV}$ for massive pions, and $C=1.04453$ for $f_{K}=110 \mathrm{MeV}$ for massive kaons (see below).

\section{Pseudoscalar pion vertex at NLO}

The pseudoscalar pion vertex can be sought at NLO using

$O_{5}(P, k)=\gamma^{5}\left(1+F_{5}(P, k)\right)+\alpha \bar{F}_{5}(P, k)+\mathcal{O}\left(\alpha^{2}\right)$

in Eq. (20) and the explicit form of the spin-valued selfenergy (16) at NLO. The result is

$$
\begin{aligned}
\bar{F}_{5}(P, k)= & K_{\pi} \bar{F}_{5}(P, k)+\rho^{4} \sum_{I+\bar{I}} \int \frac{d^{4} p}{(2 \pi)^{4}} \operatorname{Tr}_{C}\left(\Sigma_{I 1}(k, p) S_{0}(p) \gamma^{5} F_{5}(P, p) S_{0}\left(p_{-}\right) \Sigma_{I 0}\left(p_{-}, k_{-}\right)\right. \\
& \left.+\Sigma_{I 0}(k, p) S_{0}(p) \gamma^{5} F_{5}(P, p) S_{0}\left(p_{-}\right) \Sigma_{I 1}\left(p_{-}, k_{-}\right)\right)
\end{aligned}
$$

with $k_{-}, p_{-}=k-P, p-P$. The reduced kernel $K_{\pi}$ involves only the zero modes and satisfies

$$
K_{\pi} O=\rho^{4} \sum_{I, \bar{I}} \int \frac{d^{4} p}{(2 \pi)^{4}} \operatorname{Tr}_{C}\left(\Sigma_{I 0}(k, p) S_{0}(p) O S_{0}\left(p_{-}\right) \Sigma_{I 0}\left(p_{-}, k_{-}\right)\right) .
$$

Equation (35) formally defines an integral-type equation for the spin-valued operator $\bar{F}_{5}(P, k)$. The homogeneous part does not iterate due to a mismatch in chirality. In the inhomogeneous contribution, we note that most of the contributions in $\Sigma_{I 1}$ as given in Eq. (16) do not contribute due to a mismatch in chirality except for $\delta G_{I}=G_{I}-S_{0}$, and thus we have 


$$
\begin{aligned}
\bar{F}_{5}(P, k)= & \sum_{I+\bar{I}} \int \frac{d^{4} p}{(2 \pi)^{4}} \operatorname{Tr}_{C}\left(\not k \delta G_{I}(k, p) \gamma^{5} F_{5}(P, p) \psi_{0 I}\left(p_{-}\right) \frac{1}{i \sigma_{00}} \psi_{0 I}^{\dagger}\left(k_{-}\right) \not k_{-}\right) \\
& +\sum_{I+\bar{I}} \int \frac{d^{4} p}{(2 \pi)^{4}} \operatorname{Tr}_{C}\left(\not k \psi_{0 I}(k) \frac{1}{i \sigma_{00}} \psi_{0 I}^{\dagger}(p) \gamma^{5} F_{5}(P, p) \delta G_{I}\left(p_{-}, k_{-}\right) k_{-}\right) .
\end{aligned}
$$

It may be checked that the nonvanishing spin-valued structures of Eq. (37) are of the type $\gamma_{5} \not P$ and $\gamma_{5} \not k$ times invariant scalars. For estimates, we may use $\delta G_{I}$ in the Born approximation (singular gauge)

$$
\delta G_{I}(k, p) \rightarrow \frac{1}{S_{0}^{-1}+i \mathbb{A}_{I}}-S_{0} \approx-i S_{0} \mathbb{A}_{I} S_{0} \approx i S_{0}\left(\frac{\gamma^{M} \bar{\sigma}^{M N} x^{N} \rho^{2}}{\left(x^{2}+\rho^{2}\right) x^{2}}\right) S_{0} \rightarrow 4 \pi^{2} \frac{1}{\not k}\left(\gamma^{M} \bar{\sigma}^{M N} q^{N} \frac{(q \rho)^{2} K_{2}(q \rho)-2}{q^{4}}\right) \frac{1}{\not p}
$$

with $q=|p-k|$. We note that asymptotically $G_{I} \approx S_{0}$, which prompts the use of $\delta G_{I} \approx 0$ in most calculations in the random instanton model. The Born approximation allows us to go further.

\section{PION QUASIPARTON DISTRIBUTION AMPLITUDE}

The most extensively studied partonic distribution is the twist-2 pion PDA which characterizes the amplitude to find a pair of $q, \bar{q}$ with parton fraction $x, \bar{x}$ of the pion total longitudinal momentum and $x+\bar{x}=1$. The PDA is constrained by the empirical pion form factor [1] and is known at asymptotic scales to be $6 x \bar{x}$ [25]. At lower scales, there are model calculations [12-14]. Recently, a QCD lattice simulation was used to extract the pion QPDA based on the large-momentum effective theory [2] following the original suggestion in Ref. [3].

The proposed quasiparton distribution put forth in Ref. [3], translates to the pion QPDA for twist 2 as

$$
\begin{aligned}
& \tilde{\phi}_{\pi}\left(x, P_{z}\right) \\
& \quad=\frac{i}{f_{\pi}} \int \frac{d z}{2 \pi} e^{-i(x-\bar{x}) z P_{z} / 2}\left\langle\pi(p)\left|\psi^{\dagger}\left(z_{-}\right) \gamma^{z} \gamma^{5}\left[z_{-}, z_{+}\right] \psi\left(z_{+}\right)\right| 0\right\rangle
\end{aligned}
$$

where the quark fields are separated along the $z$ direction at $z_{ \pm}= \pm z / 2$ in Euclidean space, and $\left[z_{-}, z_{+}\right]$is a gauge link enforcing gauge invariance. Gauge links in Euclidean space correspond to heavy quark propagators. In the single instanton or anti-instanton background they are defined in Appendix C. Long links develop a self-energy of the form $e^{-\Delta z}$, with generically $\Delta \sim \alpha \rho$ and typically $\Delta \approx 70 \mathrm{MeV}$ [26]. Note that in the infinite-momentum limit this contribution is of order $e^{-\Delta / P_{z}} \approx 1$.

The amplitude (39) is normalized by the partially conserved axial-vector current condition

$$
\int_{-\infty}^{+\infty} d x \tilde{\phi}_{\pi}\left(x, P_{z}\right)=\frac{i}{f_{\pi} P_{z}}\left\langle\pi(p)\left|\psi^{\dagger}(0) \gamma^{z} \gamma^{5} \psi(0)\right| 0\right\rangle=1 .
$$

The pion light-cone distribution amplitude follows by taking the limit $P_{z} \rightarrow \infty$ (infinite momentum) through perturbative matching [15]. We note that $x, \bar{x}$ are in general unbound with $0 \leq x, \bar{x} \leq 1$ only expected in the infinitemomentum limit. More general properties of the QPDA were recently discussed in Ref. [17]. A more general QPDA is discussed in Appendix D.

\section{A. Planar approximation}

In the random instanton vacuum, typical planar contributions to the matrix element in Eq. (39) are illustrated in Figs. 3 and 4. It follows from the two-point-like correlator $\left\langle J_{5} \rrbracket_{5 z}\right\rangle$ where $\mathbb{J}_{5 z}$ is a point-split nonlocal source. If we set the gauge link in Eq. (39) to 1 as we argued earlier, the properly normalized result at the pion pole is

$$
\begin{aligned}
\tilde{\phi}_{\pi}\left(x, P_{z}\right)= & \lim _{P^{2} \rightarrow 0} \frac{-i}{f_{\pi} g_{\pi}} \frac{P^{2}}{P_{z}} \int \frac{d^{4} k}{(2 \pi)^{4}} \delta\left(x-\frac{1}{2}-\frac{k_{z}}{P_{z}}\right) \\
& \times \operatorname{Tr}_{C}\left(\gamma^{z} \gamma^{5} S_{1} O_{5}\left(p_{1}, p_{2}\right) S_{2}\right)
\end{aligned}
$$

where $p_{1,2}=k \pm \frac{P}{2}$ and

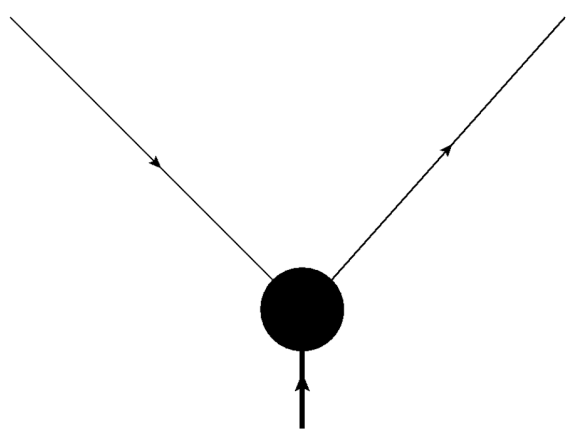

FIG. 3. Tree contribution at LO to the pion QPDA. 


$$
\begin{aligned}
& p_{1}^{2}=\left(k_{4} \pm \frac{i}{2} E_{\pi}\right)^{2}+k_{\perp}^{2}+x^{2} P_{z}^{2} \\
& p_{2}^{2}=\left(k_{4} \mp \frac{i}{2} E_{\pi}\right)^{2}+k_{\perp}^{2}+\bar{x}^{2} P_{z}^{2}
\end{aligned}
$$

with $E_{\pi}=P_{z}$. We note that Eq. (41) is of order $\alpha^{0}$ since the trace part is of order $\alpha$ and $f_{\pi} \sim \alpha$ from Eq. (32). Specifically, using the pseudoscalar vertex at NLO [Eq. (34)], we have at the pion pole



FIG. 4. Star contribution at LO to the pion QPDA.

$$
\begin{aligned}
\tilde{\phi}_{\pi}\left(x, P_{z}\right) \approx & \lim _{P^{2} \rightarrow 0} \frac{-i}{g_{\pi} f_{\pi}} \frac{P^{2}}{P_{z}} \int \frac{d^{4} k}{(2 \pi)^{4}} \delta\left(x-\frac{1}{2}-\frac{k_{z}}{P_{z}}\right) \operatorname{Tr}_{C}\left(\gamma^{z} \gamma^{5} \frac{1}{\not p_{1}} \gamma^{5} F_{5}\left(p_{1}, p_{2}\right) \frac{i \alpha \sigma_{0}\left(p_{2}\right)}{p_{2}^{2}}\right) \\
& +\lim _{P^{2} \rightarrow 0} \frac{-i}{g_{\pi} f_{\pi}} \frac{P^{2}}{P_{z}} \int \frac{d^{4} k}{(2 \pi)^{4}} \delta\left(x-\frac{1}{2}-\frac{k_{z}}{P_{z}}\right) \operatorname{Tr}_{C}\left(\gamma^{z} \gamma^{5} \frac{i \alpha \sigma_{0}\left(p_{1}\right)}{p_{1}^{2}} \gamma^{5} F_{5}\left(p_{1}, p_{2}\right) \frac{1}{\not p_{2}}\right) \\
& +\lim _{P^{2} \rightarrow 0} \frac{-i}{g_{\pi} f_{\pi}} \frac{P^{2}}{P_{z}} \int \frac{d^{4} k}{(2 \pi)^{4}} \delta\left(x-\frac{1}{2}-\frac{k_{z}}{P_{z}}\right) \operatorname{Tr}_{C}\left(\gamma^{z} \gamma^{5} \frac{1}{\not p_{1}} \alpha \bar{F}_{5}\left(p_{1}, p_{2}\right) \frac{1}{\not p_{2}}\right)
\end{aligned}
$$

where the trace is now over color-spin. Inserting the pseudoscalar vertices at NLO [Eqs. (25) and (37)] into Eq. (44) gives the leading contribution of order $\alpha^{0}$ to the QPDA

$$
\begin{aligned}
\tilde{\phi}_{\pi}\left(x, P_{z}\right) \approx & -\frac{4 N_{c}}{f_{\pi}^{2}} \int \frac{d^{4} k}{(2 \pi)^{4}} \delta\left(x-\frac{1}{2}-\frac{k_{z}}{P_{z}}\right)\left(M\left(p_{1}\right) M\left(p_{2}\right)\right)^{\frac{1}{2}}\left(\frac{\bar{x} M\left(p_{1}\right)+x M\left(p_{2}\right)}{p_{1}^{2} p_{2}^{2}}\right) \\
& +\lim _{P^{2} \rightarrow 0} \frac{\alpha}{g_{\pi} f_{\pi} \sigma_{00}} \frac{P^{2}}{P_{z}} \sum_{I+I} \int \frac{d^{4} k d^{4} q}{(2 \pi)^{8}} \delta\left(x-\frac{1}{2}-\frac{k_{z}}{P_{z}}\right) \\
& \times\left(\operatorname{Tr}_{C}\left(\gamma^{z} \gamma^{5} \delta G_{I}\left(p_{1}, q_{1}\right) \gamma^{5} F_{5}\left(q_{1}, q_{2}\right) \psi_{0 I}\left(q_{2}\right) \psi_{0 I}^{\dagger}\left(p_{2}\right)\right)\right. \\
& \left.+\operatorname{Tr}_{C}\left(\gamma^{z} \gamma^{5} \psi_{0 I}\left(p_{1}\right) \psi_{0 I}^{\dagger}\left(q_{1}\right) \gamma^{5} F_{5}\left(q_{1}, q_{2}\right) \delta G_{I}\left(q_{2}, p_{2}\right)\right)\right)
\end{aligned}
$$

with $\quad p_{1,2}=k \pm P / 2, \quad q_{1,2}=q \pm P / 2, \quad$ and $\quad E_{\pi}=\left(P_{z}^{2}+\right.$ $\left.m_{\pi}^{2}\right)^{\frac{1}{2}} \rightarrow P_{z}$ in the chiral limit. The first contribution involves only the zero modes, while the second contribution involves the cross contribution from zero modes and nonzero modes. With the help of the axial Ward identity, we have checked that to order $\alpha^{0}$, Eq. (45) with the link modification (see below) is properly normalized,

$$
\int_{-\infty}^{+\infty} d x \tilde{\phi}_{\pi}\left(x, P_{z}\right)=1 .
$$

\section{B. QPDA and PDA at LO}

\section{Zero-mode contribution}

The first contribution in Eq. (45) can be readily evaluated in singular gauge. It is solely due to the zero modes. We note that for $k_{4}=0$ a pair of poles satisfying $k_{\perp}^{2}+x^{2} P_{z}^{2}=$ $\frac{1}{4} E_{\pi}^{2}$ pinch the real $k_{4}$ integration line. To address the pinch, we rotate to Minkowski space $k_{4} \rightarrow i k_{4}$, shift $k_{4} \rightarrow k_{4}+$ $\left(x-\frac{1}{2}\right) P_{z}$ and carry out the $k_{z}$ integration to have

$$
\begin{aligned}
\tilde{\phi}_{\pi}^{0}(x) \approx & \lim _{P_{z} \rightarrow \infty} \frac{-4 i N_{c}}{f_{\pi}^{2}} \int \frac{d k_{4} d^{2} k_{\perp}}{(2 \pi)^{4}}\left(M\left(y_{1}\right) M\left(y_{2}\right)\right)^{\frac{1}{2}} \\
& \times\left(\frac{\bar{x} M\left(y_{1}\right)+x M\left(y_{2}\right)}{y_{1}^{2} y_{2}^{2}}\right)
\end{aligned}
$$

with

$$
\begin{aligned}
& y_{1}^{2}=-k_{4}\left(k_{4}+2 x P_{z}\right)+k_{\perp}^{2}-i \epsilon, \\
& y_{2}^{2}=-k_{4}\left(k_{4}-2 \bar{x} P_{z}\right)+k_{\perp}^{2}-i \epsilon .
\end{aligned}
$$

The integrand in Eq. (47) involves massless poles and also square-root branch points through the running mass (see below). The $k_{4}$ integration can be carried out by contour integration. The poles are located at

$$
\begin{aligned}
& k_{4 \pm}=-x P_{z} \pm \sqrt{x^{2} P_{z}^{2}+k_{\perp}^{2}-i \epsilon} \\
& \bar{k}_{4 \pm}=+\bar{x} P_{z} \pm \sqrt{\bar{x}^{2} P_{z}^{2}+k_{\perp}^{2}-i \epsilon} .
\end{aligned}
$$


The pair $k_{4_{-}}, \bar{k}_{4+}$ moves to infinity at large momentum $P_{z} \rightarrow \infty$ and will be ignored (their contribution is exponentially small), while the pair

$$
\begin{aligned}
& k_{4+} \approx \frac{k_{\perp}^{2}-i \epsilon}{2 x P_{z}}, \\
& \bar{k}_{4-} \approx \frac{k_{\perp}^{2}-i \epsilon}{-2 \bar{x} P_{z}}
\end{aligned}
$$

approaches the real axis, on opposite sides for $x \bar{x} \geq 0$ and the same side for $x \bar{x}<0$. In the absence of the cuts, the QPDA has support only for $x \bar{x} \geq 0$ after pole closing. To proceed further, we need to address the cuts.

\section{Unmodified effective mass at large $\boldsymbol{P}_{z}$}

In singular gauge, the running mass $M\left(y_{1,2}\right)$ at $\mathrm{LO}$ in Eq. (12) is given in terms of modified Bessel functions $I, K$ [Eq. (A1)]. When expressed in integral form, $I$ and $K$ exhibit $\left(y_{1,2}^{2}\right)^{\frac{1}{2}}$ branch points. Note that the branch points are very explicit in regular gauge with Eq. (A3)

$$
M\left(y_{1,2}\right) \approx e^{-2 \rho\left(y_{1,2}^{2}\right)^{\frac{1}{2}}} .
$$

We choose the branch cut for the square-root function $\left(\left(a-k_{4}\right)\left(b+k_{4}\right)\right)^{\frac{1}{2}}$ to be along the negative imaginary axis such that at large $k_{4}>0$ the value of the square root equals $-i$. The contour deformation of the $k_{4}$ integral into the upper half-plane, guarantees the positivity of the real part of $y_{1,2}$ and thus the decay of $M\left(y_{1,2}\right)$ asymptotically. For $x \bar{x}>0$ the contribution from the poles is purely real, while for $x \bar{x}<0$ their contribution is complex. To ensure $x \leftrightarrow \bar{x}$ symmetry of the PDA after contour integration, the branch cuts have to be arranged symmetrically for the $x$ and $\bar{x}$ contributions in Eq. (47). With this in mind, the result for the pion distribution amplitude at LO is $\left[k_{\perp} \geq M(0)\right]$

$$
\begin{aligned}
\phi_{\pi}^{0}(x) \approx & \frac{2 N_{c} M^{2}(0)}{f_{\pi}^{2}} \int \frac{d^{2} k_{\perp}}{(2 \pi)^{3}} \frac{1}{k_{\perp}^{2}}\left(\theta(x \bar{x})\left(\bar{x} M_{\perp}^{\frac{1}{2}}+x M_{\perp}^{\frac{1}{2}}\right)\right. \\
& \left.+\theta(-\bar{x}) \bar{x}\left(M_{\perp}^{\frac{1}{2}}-\bar{M}_{\perp}^{\frac{3}{2}}\right)+\theta(-x) x\left(M_{\perp}^{\frac{1}{2}}-\bar{M}_{\perp}^{\frac{3}{2}}\right)\right)
\end{aligned}
$$

with

$$
\begin{array}{lrl}
M_{\perp}=\frac{M\left(k_{\perp} / \sqrt{x}\right)}{M(0)}, & M_{\perp}=\frac{M\left(k_{\perp} / \sqrt{\bar{x}}\right)}{M(0)}, \\
\bar{M}_{\perp}=\frac{M\left(-i k_{\perp} / \sqrt{x}\right)}{M(0)}, & \bar{M}_{\perp}=\frac{M\left(-i k_{\perp} / \sqrt{\bar{x}}\right)}{M(0)}
\end{array}
$$

followed by the replacement $M(0) \rightarrow M(k)$ in the pion decay constant (32), to guarantee the normalization (46).
In the physical region $x \bar{x}>0$, Eq. (51) can be evaluated in closed form since the integrand is a total derivative,

$$
\begin{aligned}
\phi_{\pi}^{0}(x) & \approx \frac{2 N_{c} M^{2}(0)}{(2 \pi)^{2} f_{\pi}^{2}}\left(\bar{x} F\left(\frac{\rho M(0)}{2 \sqrt{x}}\right)+x F\left(\frac{\rho M(0)}{2 \sqrt{\bar{x}}}\right)\right) \\
& \rightarrow \frac{1}{\ln \left(\frac{C}{\rho M(0)}\right)}\left(\bar{x} F\left(\frac{\rho M(0)}{2 \sqrt{x}}\right)+x F\left(\frac{\rho M(0)}{2 \sqrt{\bar{x}}}\right)\right) .
\end{aligned}
$$

The rightmost relation follows from the leading-logarithm approximation for the pion decay constant, with $\rho M(0) \sim \alpha$ and

$$
F(z) \equiv I_{1}(z) K_{1}(z)-I_{0}(z) K_{0}(z) .
$$

The infrared sensitivity of the PDA follows from the enforcement of the power counting as we noted earlier. It matches the infrared sensitivity of the squared pion decay constant as given in Eq. (32), and cancels in the ratio after regulation $k_{\perp} \geq M(0)$ as we indicated earlier. To logarithmic accuracy, the PDA simplifies to

$$
\phi_{\pi}^{0}(x) \rightarrow \theta(x \bar{x})
$$

with support only in the physical range and unit normalization. In this deep infrared regime, the pion is composed democratically of partonic quarks in the range $0 \leq x \leq 1$ including the end points.

For finite-size instantons, the form factors $M_{\perp, \perp}$ cause the PDA to vanish at the end points $x=0,1$ as initially noted in Ref. [13], but it otherwise develops spurious contributions in the nonphysical region $x \bar{x}<0$ with real and imaginary parts. We recall that in the physical region with $x \bar{x} \geq 0$, the running mass involves a real combination of the modified Bessel functions $I, K$ as in Eq. (A1), and a complex combination of the cylindrical Bessel functions $J, N$ for $x \bar{x}<0$ in the unphysical regions where momentum is conserved $\left(P_{z}=x P_{z}+\bar{x} P_{z}\right)$ but energy is not $\left(\left|P_{z}\right| \neq\left|x P_{z}\right|+\left|\bar{x} P_{z}\right|\right)$.

Current lattice simulations of the quasiparton distributions [16] exhibit finite contributions outside the physically allowed $x$ support. However, they are vanishingly small at large momentum $P_{z}$. These spurious contributions are related to the transversality of the pion distribution in the QCD instanton vacuum. They do not arise in the $1 / N_{c}$ analysis in two dimensions [27]. We now show how to remove them approximately, without affecting the power counting in $\alpha$ at LO, and therefore gauge and chiral symmetry.

\section{Modified effective mass at large $\boldsymbol{P}_{z}$}

At large $P_{z}$, an approximative way to eliminate the spurious contributions without affecting the power counting in $\alpha$, is through the substitution $M(y) \rightarrow M\left(k_{\perp}\right)$, which removes explicit $k_{4}$ dependence at the integrand level. It is 
cut-free and restricts the final $k_{\perp}$ integration to the expected physical range $M(0) \leq k_{\perp} \leq 1 / \rho$. Unfortunately, this substitution fails at the end points $x, \bar{x}=0$. To see this, we recall that for fixed $k_{\perp}$, the contribution to the QPDA follows from each of the two poles in Eqs. (49)-(50) with at large $P_{z}$

$$
\begin{aligned}
& \left(y_{1}^{2}=0: y_{2}^{2}=2 k_{4} P_{z} \approx \frac{k_{\perp}^{2}}{x}\right) \quad \text { and } \\
& \left(y_{2}^{2}=0: y_{1}^{2}=-2 k_{4} P_{z} \approx \frac{k_{\perp}^{2}}{\bar{x}}\right) .
\end{aligned}
$$

When a quark (antiquark) goes on mass shell the antiquark (quark) virtuality becomes parametrically large at the end points $x, \bar{x}=0$. Say that $x, \bar{x} \approx \alpha^{2} \ll 1$ at the end points; then the $k_{\perp}$ integration range at each of the poles is vanishingly small with $M(0) \leq k_{\perp} \approx \alpha / \rho \approx M(0)$, causing the PDA to vanish. In contrast, when $x, \bar{x} \approx \alpha^{0}$ away from the end points, the $k_{\perp}$ integration range is large with $M(0) \leq k_{\perp} \leq 1 / \rho$ in line with the leading-logarithmic approximation and power counting in Eq. (53).

A simple modification of the induced effective quark mass (13) at large $P_{z}$, that enforces these observations without upsetting the power counting in $\alpha$, that is commensurate with Eq. (56) with manifest $x \leftrightarrow \bar{x}$ symmetry and free of spurious contributions, is

$$
\begin{aligned}
M(y) \rightarrow M\left(\frac{k_{\perp}}{\lambda \sqrt{|x \bar{x}|}}\right) & =M(0)\left(\left|z\left(I_{0} K_{0}-I_{1} K_{1}\right)^{\prime}\right|^{2}\right)_{z=\frac{\rho k_{\perp}}{2 \lambda_{\pi} \sqrt{|x \bar{x}|}}} \\
& \equiv M(0)\left(\left|z F^{\prime}(z)\right|^{2}\right)_{z=\frac{\rho k_{\perp}}{2 \lambda \sqrt{|x \bar{x}|}}}
\end{aligned}
$$

where $\lambda_{\pi} \approx \alpha^{0}$ is a parameter of order 1 , which is fixed by normalizing the PDA. We note that the PDA is normalized in power counting at $\mathrm{LO}$ for the unmodified effective mass.
With this in mind, the closed-form PDA at LO following from the large- $P_{z}$ limit is $\left[k_{\perp} \geq M(0)\right]$

$$
\begin{aligned}
\phi_{\pi}^{0}(x) & \rightarrow \frac{2 N_{c}}{f_{\pi}^{2}} \int \frac{d^{2} k_{\perp}}{(2 \pi)^{3}} \frac{\theta(x \bar{x})}{k_{\perp}^{2}} M^{2}\left(k_{\perp} / \lambda_{\pi} \sqrt{x \bar{x}}\right) \\
& \rightarrow \frac{\theta(x \bar{x})}{\ln \left(\frac{C}{\rho M(0)}\right)} \int_{\rho M(0) / 2 \lambda \sqrt{x \bar{x}}}^{\infty} d z z^{3} F^{\prime 4}(z) .
\end{aligned}
$$

Equation (58) is similar to Eq. (53), but with no spurious contributions! For $x, \bar{x} \approx \alpha^{2}$, the effective quark mass in Eq. (58) is probed at virtualities larger than $1 / \rho$, which is still justified by noting the agreement of the effective quark mass with the lattice data at large momenta in Fig. 2.

\section{Nonzero-mode contribution}

The nonzero-mode contributions in Eq. (45) do not vanish at finite $P_{z}$, but are in general small due to the fact that at short distances $G_{I} \approx S_{0}$ or $\delta G_{I} \approx 0$ (UV limit), a standard approximation in the random instanton model. For an estimate of their contribution, we may use the Born approximation (38) in Eq. (45). A close inspection shows that the ensuing color-spin traces are short of the binary pole structure $1 /\left(y_{1}^{2} y_{2}^{2}\right)$ which is required for 1$)$ a finite contribution as $P_{z} \rightarrow \infty$, and 2) a finite contribution for $x \bar{x} \geq 0$. In this approximation, the nonzero modes do not contribute to the PDA as $P_{z} \rightarrow \infty$.

A more explicit evaluation of the nonzero modes in Eq. (45) follows from the observation that after analytical continuation the external quark lines are put on mass shell. The ensuing contribution to Eq. (45) can be worked out in closed form. Using the modified cutoff, and the brief definitions of the mass-shell conditions in Appendix E, the result is

$$
\phi_{\pi}^{\otimes}(x) \approx \lim _{P_{z} \rightarrow \infty} \frac{\alpha}{f_{\pi}^{2}} \frac{(\sqrt{2} \pi \rho)^{2}}{\sigma_{00} P_{z}} \int \frac{d^{2} k_{\perp}}{(2 \pi)^{3}} M\left(k_{\perp} / \lambda_{\pi} \sqrt{x \bar{x}}\right) \operatorname{Tr}\left(\sigma^{z}\left(\left(\overline{\mathbb{F}}\left(P, k_{2}\right)-\overline{\mathbb{F}}\left(P, k_{1}\right)\right)-\left(\mathbb{F}\left(P, k_{2}\right)-\mathbb{F}\left(P, k_{1}\right)\right)\right) .\right.
$$

Here $k_{1,2}=k \pm P / 2$ with $k_{1,2}^{2} \approx 0$ in the large- $P_{z}$ limit and $P^{2}=0$ on mass shell. The first contribution is from the instanton and the second contribution is from the anti-instanton in the bracket. The form factors are

$$
\begin{aligned}
& \mathbb{F}(P, p)=\frac{\sigma_{z} \bar{p}+p \sigma_{z}}{2 p \cdot P} f\left(\rho \sqrt{P^{2}}\right)+\left(\frac{\sigma_{z}(\bar{P}+\bar{p})+(P+p) \sigma_{z}}{(P+p)^{2}}-\frac{\sigma_{z} \bar{p}+p \sigma_{z}}{2 p \cdot P}\right) f\left(\rho \sqrt{(p+P)^{2}}\right), \\
& \overline{\mathbb{F}}(P, p)=\frac{\sigma_{z} p+\bar{p} \sigma_{z}}{2 p \cdot P} f\left(\rho \sqrt{P^{2}}\right)+\left(\frac{\sigma_{z}(P+p)+(\bar{P}+\bar{p}) \sigma_{z}}{(\bar{P}+\bar{p})^{2}}-\frac{\sigma_{z} p+\bar{p} \sigma_{z}}{2 p \cdot P}\right) f\left(\rho \sqrt{(p+P)^{2}}\right)
\end{aligned}
$$

with $f(z)=z K_{1}(z)-1$. Throughout, the Weyl notation $p=p_{\mu} \sigma^{\mu}, \bar{p}=p_{\mu} \bar{\sigma}^{\mu}$ etc. is used with $\sigma^{\mu}=(1, \vec{\sigma})$ and $\bar{\sigma}^{\mu}=(1,-\vec{\sigma})$. The finite contribution in Eq. (60) when inserted into Eq. (59) cancels out. The nonzero-mode contribution (59) to the PDA vanishes at LO. 


\section{Massive pion and kaon}

The explicit breaking of chiral symmetry by light quark masses $u, d, s$ is understood in the QCD instanton vacuum, with the masses for the pion and kaon at LO obeying the Gell-Mann-Oakes-Renner (GOR) relation [5-7]. In our case, this can be explicitly checked to hold in power counting. For a finite current mass $m$ a rerun of the arguments leading to the effective quark mass in Eq. (12) yields

$$
\begin{aligned}
M(p, m) & =\frac{\alpha\left|p \varphi^{\prime}(p)\right|^{2}}{\left(2\left\|q \varphi^{\prime 2}\right\|^{2}+\frac{m^{2}}{4 \alpha^{2}}\right)^{\frac{1}{2}}+\frac{m}{2 \alpha}}+m \\
& \equiv \frac{M(p, 0)}{\left(1+\xi^{2}\right)^{\frac{1}{2}}+\xi}+m
\end{aligned}
$$

with the mass parameter

$$
\xi=\frac{m M(0,0) \rho^{2}}{8 \pi^{2} \kappa} .
$$

For light quarks $M(0) \equiv M(0,0) \approx 386 \mathrm{MeV}$ and $\rho \approx$ $1 /(631 \mathrm{MeV})$. For massive quarks we find $M(0,5) \approx$ $383.7 \mathrm{MeV} \approx M(0)$ and $M(0,150) \approx 372.6 \mathrm{MeV} \approx M(0)$ for the up/down and strange quarks respectively. The effective quark mass is almost unchanged. As a result, the pseudoscalar decay constants for the pion and kaon are about the same at LO for massive quarks.

For the massive case, the integral equation (25) for the pseudoscalar meson vertex holds with the substitution

$$
\sigma_{00} \rightarrow\left(2\left\|q \varphi^{\prime 2}\right\|^{2}+\frac{m^{2}}{4 \alpha^{2}}\right)^{\frac{1}{2}}+\frac{m}{2 \alpha} \approx \sqrt{2}\left\|q \varphi^{\prime 2}\right\|+\frac{m}{2 \alpha} .
$$

As a result the mass-shell vertex (27) at LO changes to

$$
\begin{aligned}
& \left(i O_{5}(P, k)\right)_{P^{2} \approx-m_{P}^{2}} \\
& \quad \approx \frac{\sqrt{N_{c}}}{f_{P}} \sqrt{M(k)}\left(\frac{i \gamma_{5}}{P^{2}+m_{P}^{2}}\right) \sqrt{M(k-P)}
\end{aligned}
$$

with $f_{P} \approx f_{\pi}$ and $m_{P}^{2} \approx i 2 m\left\langle\psi^{\dagger} \psi\right\rangle$ as expected. Note that in Eq. (64) both $f_{P}$ and $M(k)$ are found to be unaffected by the current mass $m$ at the meson pole at LO. The latter only shifts the meson pole in agreement with the GOR relation.

The ensuing PDA for massive pseudoscalars simplifies to $\mathrm{LO}$

$$
\begin{aligned}
\phi_{P}^{0}(x) \approx & \frac{2 N_{c} M^{2}(0)}{f_{P}^{2}} \int \frac{d^{2} k_{\perp}}{(2 \pi)^{3}} \frac{1}{k_{\perp}^{2}-x \bar{x} m_{P}^{2}} \\
& \times\left(\theta(x \bar{x})\left(\bar{x} M_{\perp}+x M_{\perp}\right)+\theta(-\bar{x}) \bar{x}\left(M_{\perp}-M_{\perp}\right)\right. \\
& \left.+\theta(-x) x\left(M_{\perp}-M_{\perp}\right)\right)
\end{aligned}
$$

with the same cutoff $k_{\perp} \geq M(0)$ for light quarks $u, d, s$. For comparison, the result for the modified effective quark mass (57) is

$\phi_{P}^{0}(x) \rightarrow \frac{2 N_{c}}{f_{P}^{2}} \int \frac{d^{2} k_{\perp}}{(2 \pi)^{3}} \frac{\theta(x \bar{x})}{k_{\perp}^{2}-x \bar{x} m_{P}^{2}} M^{2}\left(k_{\perp} / \lambda_{P} \sqrt{x \bar{x}}\right)$.

In Fig. 5 we show the pion PDA (66) at LO for varying $\rho$ but fixed $M(0)=386 \mathrm{MeV}$ (solid curves) in comparison to the asymptotic result of $6 x \bar{x}$ [25] (dashed curve). We have set $f_{\pi}=93 \mathrm{MeV}$ and $m_{\pi}=135 \mathrm{MeV}$ and fixed $\lambda_{\pi}=$ 3.41894 for the overall normalization of the PDA with the modified effective mass. (No such modification is needed for the unmodified effective quark mass). The result at this low renormalization scale $Q_{0}=1 / \rho$ is remarkably close to the QCD asymptotic result of $6 x \bar{x}$ [25]. The single $q \bar{q}$ component of the pion wave function is well described in the random instanton vacuum (RIV) in the planar approximation at LO. Since the constituent mass $M(0) \approx$ $M(0,5) \approx M(0,150)$ is almost unchanged for $u, d, s$, the kaon PDA is almost undistinguishable from the pion PDA at LO.

Our result for the pion PDA at LO is similar to the one obtained originally in Ref. [13] using time-like arguments with a modified dipole effective quark mass with very different analytical properties. It is overall analogous to the one derived from modified holographic models [9]. As $\rho \rightarrow 0$, and the cutoff is removed, the pion PDA asymptotes to middle solid red curve in Fig. 5 which is close to the normalized step function $\theta(x \bar{x})$. The same result was noted for chiral quark models with point interactions [11,12], and some bound-state resummations [10].

In Fig. 6 we compare our result for the pion PDA (RIV, red solid line) to the recently generated pion PDA (blue band), using lattice simulations using the large momentum effective theory (LaMET) [2]. The QCD asymptotic result (black dashed curve) is again shown for comparison.

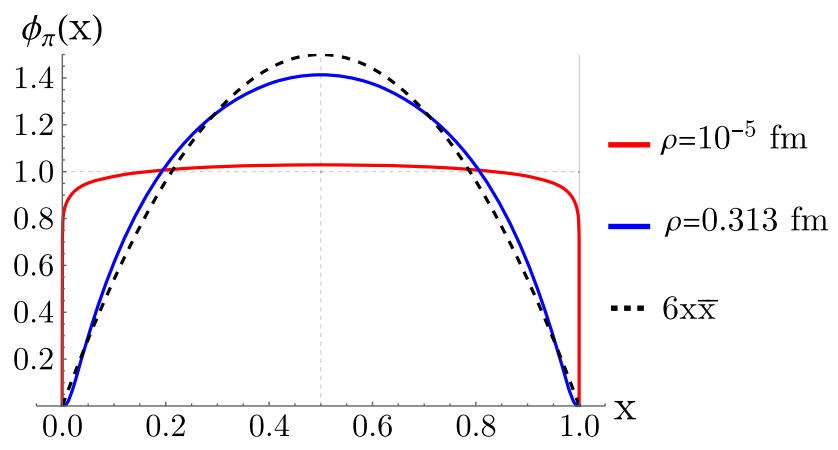

FIG. 5. Pion PDA (66) for varying instanton size $\rho$ but fixed $M(0)=386 \mathrm{MeV}$ (solid curves) in comparison to the asymptotic result of $6 x \bar{x}$ [25] (dashed curve). 


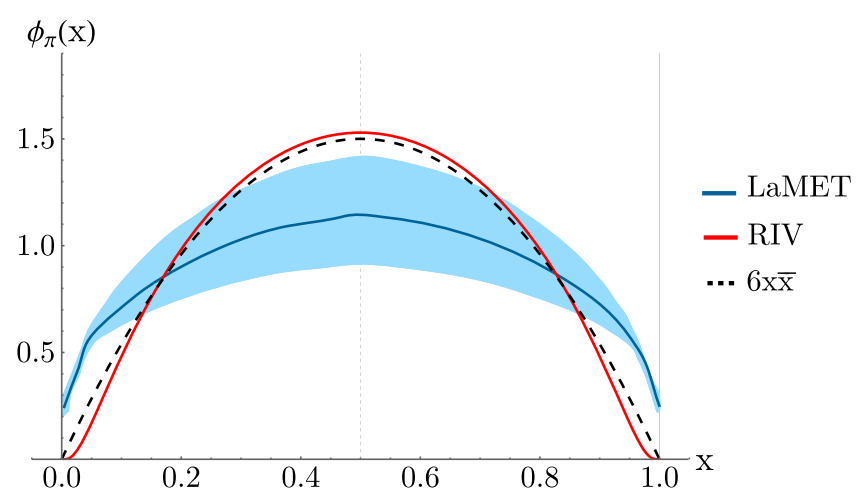

FIG. 6. Pion PDA from the random instanton model (RIV, solid red curve) (66) and asymptotic QCD (dashed black curve) [25] in comparison to the lattice simulations using LaMET (blue wide band) [2].

\section{QCD evolution of pion PDA}

The pion PDA (66) is defined at a low renormalization scale set by the instanton size $Q_{0}=1 / \rho=631 \mathrm{GeV}$. Assuming factorization, its form at higher renormalization scales follows from a QCD kernel evolution equation (ERBL). Its closed-form solution in the form of Gegenbauer polynomials was given in Ref. [25]. More specifically, using Eq. (58) as an initial condition, the ERBL-evolved pion PDA is [25]

$\phi_{\pi}(x, Q)=6 x \bar{x} \sum_{n-\text { even }} a_{n}\left(Q_{0}\right)\left(\frac{\alpha_{s}(Q)}{\alpha_{s}\left(Q_{0}\right)}\right)^{\gamma_{n} / \beta_{0}} C_{n}^{\frac{3}{2}}(x-\bar{x})$

with the initial coefficients

$a_{n}\left(Q_{0}\right)=\frac{2(2 n+3)}{3(n+1)(n+2)} \int_{0}^{1} d y C_{n}^{\frac{3}{2}}(y-\bar{y}) \phi_{\pi}^{0}(y)$.

Here $\alpha_{s}(Q)=4 \pi / \beta_{0} \ln \left(Q^{2} / \Lambda^{2}\right)$ is the one-loop running QCD coupling with $\beta_{0}=\frac{11}{3} N_{c}-\frac{2}{3} N_{f}$ and $\Lambda=250 \mathrm{MeV}$ ( $\overline{\mathrm{MS}}$ scheme). The $\gamma_{n}$ are pertinent anomalous dimensions

$$
\gamma_{n}=C_{F}\left(1+4 \sum_{k=2}^{n+1} \frac{1}{k}-\frac{2}{(n+1)(n+2)}\right)
$$

with the Casimir $C_{F}=\left(N_{c}^{2}-1\right) / 2 N_{c}$. Since $\gamma_{0}=1$ and $\gamma_{n}>0$, it follows that Eq. (67) asymptotes to $6 x \bar{x}$ with $a_{0}\left(Q_{0}\right)=1$ for $Q \rightarrow \infty$ as illustrated in Fig. 5 .

In Fig. 7 we show the ERBL-evolved pion PDA (58) at $Q=2 \mathrm{GeV}$ as a green solid curve (RIV), which is in good agreement with the empirical pion PDA (blue data points) extracted from dijet data from the E791 Collaboration [28] at the same scale. For comparison, we also show the chiral

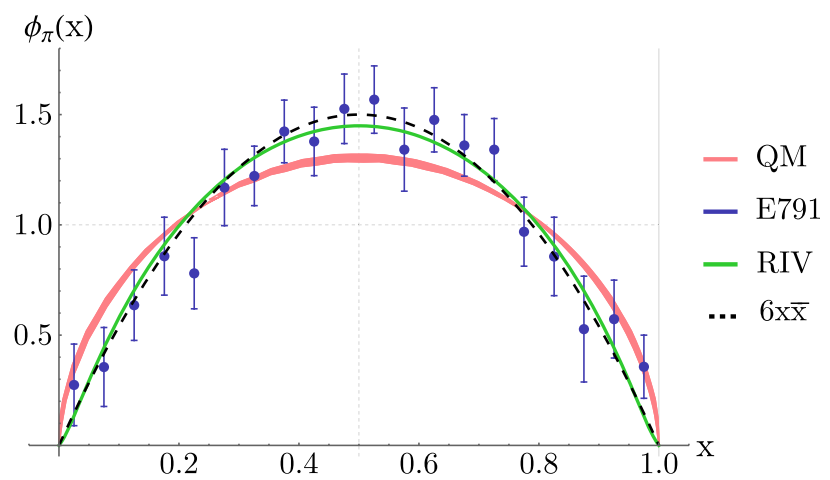

FIG. 7. Pion PDA from the random instanton model evolved to $Q=2 \mathrm{GeV}$ (RIV, solid red curve) (58), the quark model QM@2 GeV (QM, solid pink band) [12], and asymptotic QCD (dashed black curve) [25] in comparison to the E791 dijet data [28].

quark model evolved PDA to $Q=2 \mathrm{GeV}$ as a solid pink band $(\mathrm{QM})$ [12] and the asymptotic QCD result [25]. Again, since $M(0)$ does not change much for massive pions and kaons, the ERBL-evolved kaon PDA is indistinguishable from its evolved pion counterpart at $\mathrm{LO}$ in the present analysis.

\section{PION QUASIPARTON DISTRIBUTION FUNCTION}

In this section we show how to resum the planar contributions to the three-point functions in general. We then apply the results to the derivation of the pion valence quasiparton distribution function to LO. Since this distribution obeys charge and momentum sum rules, the enforcement of the gauge and chiral symmetry through the Ward identity is needed.

\section{A. Three-point function}

The quasiparton distribution functions involve threepoint functions with one of the source point-split in space. In the planar approximation, their construction follows a similar reasoning as the one developed earlier. For that, consider the general three-point function

$$
\left\langle\mathrm{O}_{1} \mathrm{O}_{3} \mathrm{O}_{2}\right\rangle
$$

where the $O$ 's are resummed and colorless local or quasilocal fermionic bilinears defined as

$$
O_{a b}=\operatorname{Tr}_{C}\left(S_{\gamma \beta} O_{\beta \alpha} S_{\alpha \delta} T_{a \gamma ; \delta b}\right)
$$

and are spin-flavor valued in general. In the planar approximation, the leading contributions to Eq. (70) are 


$$
\left\langle O_{1} O_{3} O_{2}\right\rangle=\operatorname{Tr}_{C}\left(O_{3} S O_{2} S O_{1} S\right)+\frac{N}{2 N_{c} V} \int_{I+\bar{I}} d z_{I} \operatorname{Tr}_{C}\left(O_{3} S\left(-\Sigma_{I}\right) S O_{2} S\left(-\Sigma_{I}\right) S O_{1} S\left(-\Sigma_{I}\right) S\right)
$$

The first contribution sums up all planar diagrams with no common instanton to the three quark lines as illustrated in Fig. 8. The second contribution corresponds to the planar contributions with one instanton shared by the three quark lines. Planarity implies that only one instanton is commonly shared by the three quark lines as shown in Fig. 9. For a finite gauge link $\left[z_{-}, z_{+}\right]$there is an additional contribution shown in Fig. 10 with $I, J$ referring to a double summation over distinct instantons (anti-instantons). It is readily seen that this contribution reduces to that shown in Fig. 9 when the gauge link is 1, so it will be ignored. The direct and cross contributions follow from pertinent rerouting of the momenta. The extension of these observations to the $n$-point functions is now straightforward.

\section{B. Pion QPDF and PDF at LO}

The pion QPDF can also be extracted from the equaltime correlator following Eq. (1) as suggested in Ref. [3]. In our case it follows by reduction using the pseudoscalar source. Specifically, in the chiral limit we have

$$
\begin{aligned}
\tilde{\psi}_{\pi}\left(x, P_{z}\right) & =\int \frac{d z}{2 \pi} e^{-\frac{i}{2}(x-\bar{x}) z P_{z}}\left\langle\pi(P)\left|\psi^{\dagger}\left(z_{-}\right) \gamma^{z}\left[z_{-}, z_{+}\right] \psi\left(z_{+}\right)\right| \pi(P)\right\rangle \\
& \approx \lim _{P^{2} \rightarrow 0} \frac{P^{4}}{g_{\pi}^{2}} \int \frac{d z}{2 \pi} e^{-\frac{i}{2}(x-\bar{x}) z P_{z}}\left\langle O_{5}(-P) \psi^{\dagger}\left(z_{-}\right) \gamma^{z}\left[z_{-}, z_{+}\right] \psi\left(z_{+}\right) O_{5}(P)\right\rangle .
\end{aligned}
$$

Following the previous reasoning we may approximate the gauge link as 1 in the large- $P_{z}$ limit. Using the density expansion for the pseudoscalar vertex (34) and the effective mass (16) at NLO, we can unwind Eq. (73). The result at LO is

$$
\begin{aligned}
& \tilde{\psi}_{\pi}\left(x, P_{z}\right) \\
& \approx-\lim _{P^{2} \rightarrow 0} \frac{P^{4}}{g_{\pi}^{2} P_{z}} \int \frac{d^{4} k}{(2 \pi)^{4}} \delta\left(x-\frac{1}{2}-\frac{k_{z}}{P_{z}}\right) \operatorname{Tr}_{C}\left(\gamma^{z} \frac{1}{\not k_{1}} \gamma^{5} F_{5}(P, k) \frac{1}{\not k_{2}} \gamma^{5} F_{5}(P, k) \frac{1}{\not k_{1}}\right)+\mathrm{cross} \\
& +\lim _{P^{2} \rightarrow 0} \frac{P^{4}}{g_{\pi}^{2} \sigma_{00}^{2} P_{z}} \int \frac{d^{4} k}{(2 \pi)^{4}} \frac{d^{4} q}{(2 \pi)^{4}} \frac{d^{4} p}{(2 \pi)^{4}} \delta\left(x-\frac{1}{2}-\frac{k_{z}}{P_{z}}\right) \operatorname{Tr}_{C}\left(\gamma^{z} \psi_{0 I}\left(k_{1}\right) \psi_{0 I}^{\dagger}\left(q_{1}\right) \gamma^{5} F_{5}(P, q) \delta G_{I}\left(q_{2}, p_{2}\right) \gamma^{5} F_{5}(P, p) \psi_{0 I}\left(p_{1}\right) \psi_{0 I}^{\dagger}\left(k_{1}\right)\right) \\
& +\lim _{P^{2} \rightarrow 0} \frac{P^{4}}{g_{\pi}^{2} \sigma_{00}^{2} P_{z}} \int \frac{d^{4} k}{(2 \pi)^{4}} \frac{d^{4} q}{(2 \pi)^{4}} \frac{d^{4} p}{(2 \pi)^{4}} \delta\left(x-\frac{1}{2}-\frac{k_{z}}{P_{z}}\right) \operatorname{Tr}_{C}\left(\gamma^{z} \psi_{0 I}\left(k_{1}\right) \psi_{0 I}^{\dagger}\left(q_{1}\right) \gamma^{5} F_{5}(P, q) \psi_{0 I}\left(q_{1}\right) \psi_{0 I}^{\dagger}\left(p_{2}\right) \gamma^{5} F_{5}(P, p) \delta G_{I}\left(p_{1}, k_{1}\right)\right) \\
& +\lim _{P^{2} \rightarrow 0} \frac{P^{4}}{g_{\pi}^{2} \sigma_{00}^{2} P_{z}} \int \frac{d^{4} k}{(2 \pi)^{4}} \frac{d^{4} q}{(2 \pi)^{4}} \frac{d^{4} p}{(2 \pi)^{4}} \delta\left(x-\frac{1}{2}-\frac{k_{z}}{P_{z}}\right) \operatorname{Tr}_{C}\left(\gamma^{z} \delta G_{I}\left(k_{1}, q_{1}\right) \gamma^{5} F_{5}(P, q) \psi_{0 I}\left(q_{2}\right) \psi_{0 I}^{\dagger}\left(p_{2}\right) \gamma^{5} F_{5}(P, p) \psi_{0 I}\left(p_{1}\right) \psi_{0 I}^{\dagger}\left(k_{1}\right)\right)
\end{aligned}
$$

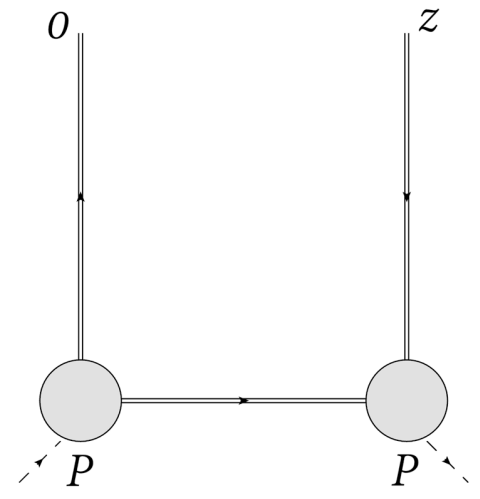

FIG. 8. Tree contribution at LO to the pion QPDF.

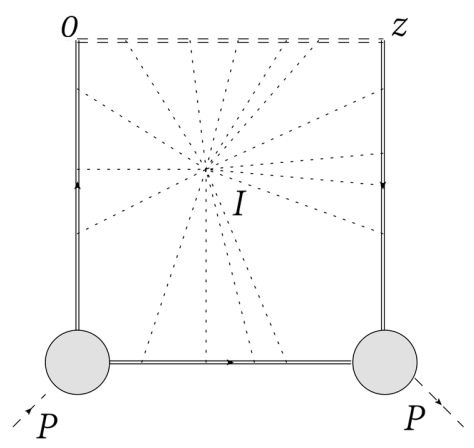

FIG. 9. Star contribution at LO to the pion QPDF. 


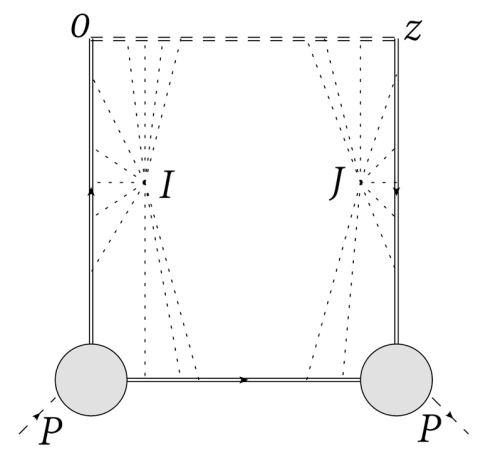

FIG. 10. Split contribution at LO to the pion QPDF.

with $k_{1,2}=k \pm \frac{P}{2}$ and so on. The summation over $I, \bar{I}$ is subsumed. The cross refers to the cross contributions (see below). All contributions are of order $\alpha^{0}$ since $g_{\pi} \sim \alpha^{0}$ and $\sigma_{00} \sim \alpha^{0}$. The first contribution involves only the zero modes. The second to fourth contributions involve the cross contributions from the zero and nonzero modes. The latter are required for the enforcement of the Ward identities in power counting, and all contributions are of the same order in $\alpha$. We note that the second contribution in Eq. (74) vanishes due to a mismatch in chirality.

\section{Nonzero-mode contribution}

An explicit evaluation of the nonzero modes in Eq. (74) is involved, but follows from the observation that after analytical continuation the external quark lines are put on mass shell as we noted earlier for the PDA. In Appendix E the rules for putting the instanton zero modes and nonzero-mode propagator on mass shell are given. The ensuing contribution to Eq. (74) can be worked out in closed form much like for the PDA. Using the modified cutoff, the result is

$$
\begin{aligned}
\psi_{\pi}^{\varnothing}(x) \approx & \lim _{P_{z} \rightarrow \infty} \frac{(\sqrt{2} \pi \rho)^{2}}{f_{\pi}^{2} \sigma_{00}^{2} P_{z}} \int \frac{d^{2} k_{\perp}}{(2 \pi)^{3}} M^{2}\left(k_{\perp} / \lambda_{\pi} \sqrt{x \bar{x}}\right) \\
& \times \operatorname{Tr}\left(\sigma^{z}\left(2 \mathbb{F}\left(0, k_{1}\right)+2 \overline{\mathbb{F}}\left(0, k_{1}\right)\right)\right.
\end{aligned}
$$

with $k_{1}=k+P / 2$ and $k_{1}^{2} \approx 0$ at large $P_{z}$. The form factors are given in Eq. (60). They are zero for the present kinematics. The nonzero-mode contribution (75) vanishes. So in the large-momentum limit the pion PDF at
LO is dominated by the zero-mode contribution which we now explicitly show.

\section{Pion and kaon PDF at LO and large $P_{z}$}

The first contribution in Eq. (74) is dominated by the pion pole. Inserting Eq. (25), carrying the spin trace, unwinding the $k_{z}$ integration and analytically continuing $k_{4} \rightarrow i k_{4}$ yields $\left[k_{\perp} \geq M(0)\right]$

$$
\begin{aligned}
\tilde{\psi}_{\pi}^{0}\left(x, P_{z}\right) \approx & \frac{4 i N_{c}}{f_{\pi}^{2}} \int \frac{d k_{4} d^{2} k_{\perp}}{(2 \pi)^{4}}\left(M\left(y_{1}\right) M\left(y_{2}\right)\left(\frac{x+\bar{x}}{y_{1}^{2} y_{2}^{2}}+\frac{x}{y_{1}^{4}}\right)\right. \\
& \left.+M\left(y_{1}\right) M\left(y_{2}^{\prime}\right)\left(-\frac{x+\bar{x}}{y_{1}^{2}\left(y_{2}^{\prime}\right)^{2}}+\frac{x}{y_{1}^{4}}\right)\right)
\end{aligned}
$$

with $y_{1,2}$ given in Eq. (48), and $y_{2}^{\prime}=k+P$ in the cross contribution. Equation (76) can be undone by pole closing. In the large- $P_{z}$ limit, the cross contribution in Eq. (76) and the contribution $1 / y_{1}^{4}$ in Eq. (76) are subleading. Using the unmodified effective quark mass (13), the result for the pion valence $\mathrm{PDF}$ at $\mathrm{LO}$ and large $P_{z}$ and in the chiral limit is

$$
\begin{aligned}
\psi_{\pi}^{0}(x) \approx & \frac{2 N_{c} M^{2}(0)}{f_{\pi}^{2}} \int \frac{d^{2} k_{\perp}}{(2 \pi)^{3}} \frac{1}{k_{\perp}^{2}}\left(\theta(x \bar{x})\left(\bar{x} M_{\perp}+x M_{\perp}\right)\right. \\
& \left.+\theta(-\bar{x}) \bar{x}\left(M_{\perp}-M_{\perp}\right)+\theta(-x) x\left(M_{\perp}-M_{\perp}\right)\right) .
\end{aligned}
$$

Note that a similar conclusion follows from the free approximation for the nonzero modes $\delta G_{I} \approx 0$, or the Born approximation (38). For comparison, the result with the modified effective quark mass (57) is

$\psi_{\pi}^{0}(x) \rightarrow \frac{2 N_{c}}{f_{\pi}^{2}} \int \frac{d^{2} k_{\perp}}{(2 \pi)^{3}} \frac{\theta(x \bar{x})}{k_{\perp}^{2}} M^{2}\left(k_{\perp} / \lambda_{\pi} \sqrt{x \bar{x}}\right) \approx \phi_{\pi}^{0}(x)$.

Away from the chiral limit, the QPDF involves several contributions that will be presented elsewhere. We have checked that the PDF limit at LO simplifies. One can split the valence PDF between the different individual quark distributions. For the unmodified quark effective mass (13), the PDF for flavor $f$ in pseudoscalar $P$ or $f / P$ is

$\psi_{f / P}^{0}(x) \approx \frac{N_{c} M^{2}(0)}{f_{P}^{2}} \int_{f} \frac{d^{2} k_{\perp}}{(2 \pi)^{3}} \frac{k_{\perp}^{2}}{\left(k_{\perp}^{2}-x \bar{x} m_{P}^{2}\right)^{2}}\left(\theta(x \bar{x})\left(\bar{x} M_{\perp}+x M_{\perp}\right)+\theta(-\bar{x}) \bar{x}\left(M_{\perp}-M_{\perp}\right)+\theta(-x) x\left(M_{\perp}-M_{\perp}\right)\right)$

while for the modified quark effective mass (57) it is

$$
\psi_{f / P}^{0}(x) \rightarrow \frac{N_{c}}{f_{P}^{2}} \int_{f} \frac{d^{2} k_{\perp}}{(2 \pi)^{3}} \frac{\theta(x \bar{x}) k_{\perp}^{2}}{\left(k_{\perp}^{2}-x \bar{x} m_{P}^{2}\right)^{2}} M^{2}\left(k_{\perp} / \lambda_{P} \sqrt{x \bar{x}}\right) .
$$




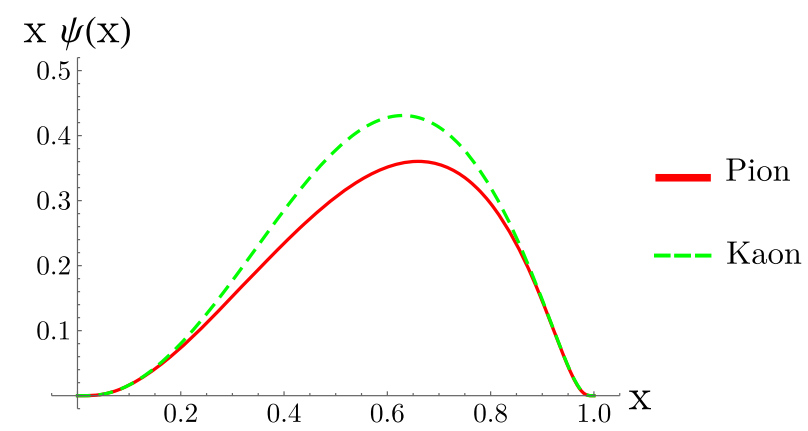

FIG. 11. Comparison of the pion (red solid) vs kaon (green dashed) valence longitudinal momentum distributions at the factorization scale $Q=Q_{0}=631 \mathrm{MeV}$.

The $f$ integration is carried out with $k_{\perp} \geq M\left(0, m_{f}\right)$, with $f=u, d$ for the pion and $f=u, s$ for the kaon. The full valence PDFs are obtained by summing the individual valence-quark PDFs, i.e., $\psi_{\pi}=\psi_{u / \pi}+\psi_{d / \pi}$, and $\psi_{K}=\psi_{u / K}+\psi_{s / K}$.

In Fig. 11 the pion and kaon valence PDFs are shown at the factorization scale $Q=Q_{0}=631 \mathrm{MeV}$ (see next section). Unlike the PDAs, there is a noticeable difference between the two PDFs. In Fig. 12 the kaon $u$ and $s$ quark distributions are shown. These two plots exhibit the extent to which flavor $\mathrm{SU}(3)$ symmetry is broken in our calculations of the integrated PDFs at LO.

\section{QCD evolution of pion PDF}

To compare the pion valence PDF in the random instanton vacuum at the inverse instanton size scale $Q_{0}=1 / \rho=631 \mathrm{MeV}$, with the measured pion PDF at higher resolution we need to evolve the pion PDF (80) to a higher scale using QCD evolution (DGLAP). The one-loop DGLAP evolution of the valence (nonsinglet/forward) pseudoscalar PDF $\psi_{P}(x, t)$ is

$$
\frac{d \psi_{P}(x, t)}{d t}=\frac{\alpha_{s}(t)}{2 \pi} \int_{x}^{1} \frac{d y}{y} P_{q q}^{(0)}\left(\frac{x}{y}\right) \psi_{P}(y, t)
$$

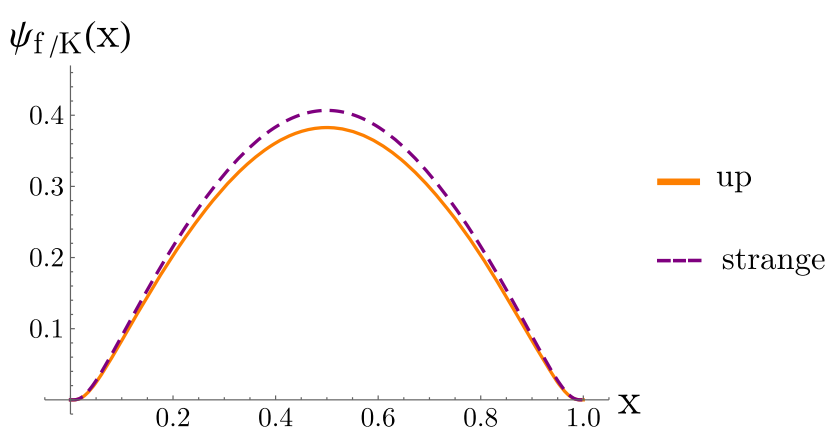

FIG. 12. Comparison of the valence kaon $u$-quark distribution (orange solid) vs $s$-quark distribution (purple dashed) at the factorization scale $Q=Q_{0}=631 \mathrm{MeV}$. with $t=\log \left(Q^{2} / \Lambda_{\mathrm{QCD}}^{2}\right)$ and $P_{q q}^{(0)}(z)$ is the one-loop nonsinglet splitting function

$$
P_{q q}^{(0)}(z)=C_{F}\left[\frac{1+z^{2}}{(1-z)_{+}}+\frac{3}{2} \delta(1-z)\right] .
$$

We numerically evolve from $t$ to $t+\Delta t$ by simple forwardEuler. We sample $\psi_{P}(x, t)$ on a uniform grid in $x$, create a spline interpolation, and evaluate the rhs of Eq. (81) to calculate $d \psi$. The consistency of the evolution is checked in two ways: first by verifying that the first few Mellin moments evolve according to the analytical result

$M_{n}(t) \equiv \int_{0}^{1} x^{n} \psi_{P}(x, t) d x=M\left(t_{0}\right)\left(\frac{\alpha_{s}(t)}{\alpha_{s}\left(t_{0}\right)}\right)^{\gamma_{n} / \beta_{0}}$

where $\gamma_{n}$ is the same as before in Eq. (69), and second by reproducing the evolution of Ref. [12] where the authors evolved a step function $\psi_{P}\left(x, t_{0}\right)=\theta(x \bar{x})$ from $Q_{0}=313 \mathrm{MeV}$ to $Q=2 \mathrm{GeV}$ with $\Lambda_{\mathrm{QCD}}=226 \mathrm{MeV}$.

In Fig. 13 we show the result for the pion longitudinal momentum distribution in our QCD RIV (77) (solid red curve) evolved to $Q^{2}=16 \mathrm{GeV}^{2}$. The data are from the E615 Collaboration (blue squares) [29], and the improved E615 data (inverse blue triangles) [30]. The Electron-Ion Collider (EIC) projection is shown by the solid orange curve [31]. All are evolved to the same $Q^{2}=16 \mathrm{GeV}^{2}$. In Fig. 14 we show the pion longitudinal distribution in the QCD RIV (solid red curve) in comparison to recent lattice results (LCSs, blue band) [32] at a higher scale $Q^{2}=27 \mathrm{GeV}^{2}$. There is good agreement at large $x$, but the RIV results fall short at low $x$. This may be a shortcoming of our planar approximation which ignores multi- $q \bar{q}$ or sea contributions to the pion wave function at low $x$. We note that for smaller size instantons $\rho \rightarrow 0$, the pion (and kaon) PDF approaches a step function, as does the PDA shown in Fig. 5. Its DGLAP evolution is more in line with the data

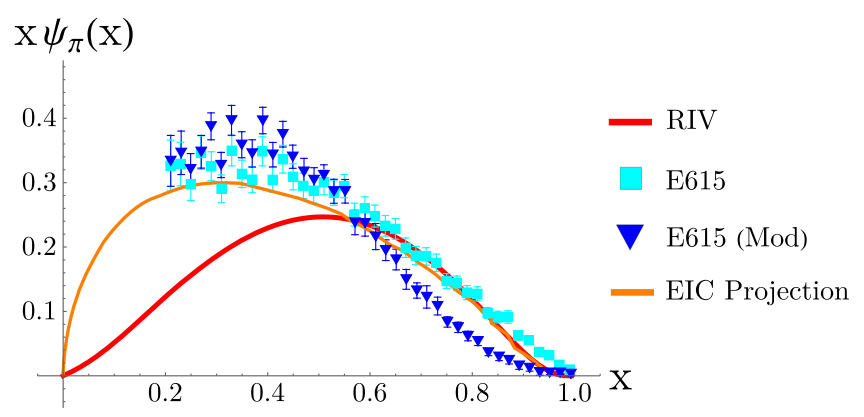

FIG. 13. Pion valence longitudinal momentum distribution in the QCD instanton vacuum RIV (solid red curve) (77), the E615 data (blue squares) [29] devolved to $Q=4 \mathrm{GeV}$ with next-toNLO DGLAP of Lan et al. [11], improved E615 data (inverse blue triangles) [30], and the EIC projection (solid orange curve) [31]. All are presented at the factorization scale $Q^{2}=16 \mathrm{GeV}^{2}$. 




FIG. 14. Pion longitudinal momentum distribution in the QCD instanton vacuum evolved to $Q^{2}=27 \mathrm{GeV}^{2}$ (RIV, solid red curve) (77) in comparison to the lattice results LCSs (blue band) at the same scale [32].

for all $x$ (specifically low $x$ where we currently see noticeable disagreement). These are the results found in Ref. [12]. However, smaller size instantons do not support the key vacuum parameters we established earlier.

\section{Pion and kaon TMD at LO and large $\boldsymbol{P}_{z}$}

Finally, we note that the integrand in Eq. (80) describes the parton TMD in a pseudoscalar $P$. However at this point we must recall Eq. (23), namely, that our actual leading-order TMD is only obtained after shifting back $k_{\perp}^{2} \rightarrow k_{\perp}^{2}+M^{2}(0)$. It follows that the TMD for the massive pion at $\mathrm{LO}$ is

$$
\begin{aligned}
\psi_{\pi}^{0}\left(x, k_{\perp}\right) \rightarrow & \frac{2 N_{c}}{f_{\pi}^{2}} \frac{1}{(2 \pi)^{3}} \frac{\theta(x \bar{x})\left(k_{\perp}^{2}+M^{2}(0)\right)}{\left(k_{\perp}^{2}+M^{2}(0)-\bar{x} x m_{\pi}^{2}\right)^{2}} \\
& \times M^{2}\left(\frac{\sqrt{k_{\perp}^{2}+M^{2}(0)}}{\lambda \sqrt{x \bar{x}}}\right)
\end{aligned}
$$

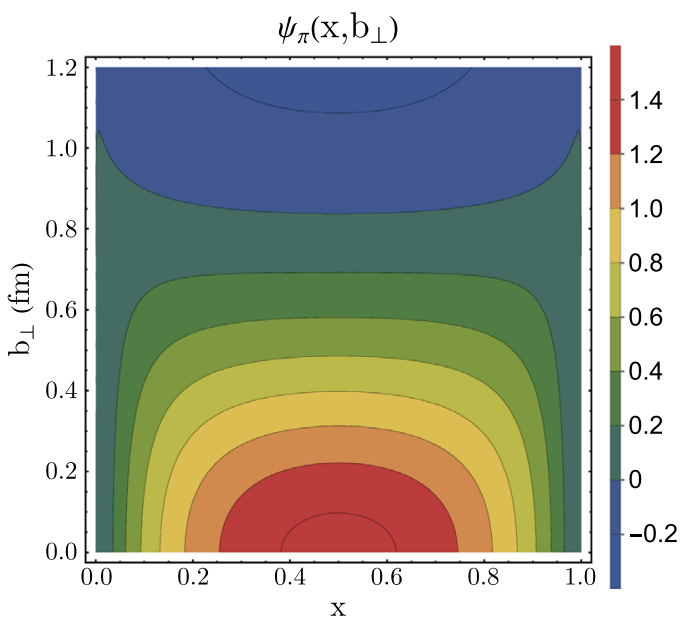

while the transverse spatial distribution is

$$
\begin{aligned}
& \psi_{\pi}^{0}\left(x, b_{\perp}\right) \\
& \qquad \rightarrow \frac{2 N_{c}}{f_{\pi}^{2}} \int \frac{d^{2} k_{\perp}}{(2 \pi)^{3}} e^{i k_{\perp} \cdot b_{\perp}} \frac{\theta(x \bar{x}) k_{\perp}^{2}}{\left(k_{\perp}^{2}-\bar{x} x m_{\pi}^{2}\right)^{2}} M^{2}\left(k_{\perp} / \lambda \sqrt{x \bar{x}}\right)
\end{aligned}
$$

with $k_{\perp} \geq M(0)$ subsumed. The leading-logarithm contribution to the TMD in the massless case is

$$
\begin{aligned}
& \psi_{\pi}^{0}\left(x, b_{\perp}\right) \\
& \qquad \rightarrow \frac{\theta(x \bar{x})}{4 \ln \left(\frac{C}{\rho M(0)}\right)} \int_{\rho M(0) / 2 \lambda \sqrt{x \bar{x}}}^{\infty} d z J_{0}\left(2 \sqrt{x \bar{x}} \frac{z b_{\perp}}{\rho}\right) z^{3} F^{\prime 4}(z) .
\end{aligned}
$$

For comparison, the massless pion TMD with the unmodified effective quark mass (13) in the physical region $x \bar{x} \geq 0$, is

$$
\begin{aligned}
\psi_{\pi}^{0}\left(x, b_{\perp}\right) \approx & \frac{N_{c} \rho^{2} M^{2}(0)}{2 f_{\pi}^{2}} \int_{M(0)}^{\infty} \frac{k_{\perp} d k_{\perp}}{(2 \pi)^{2}} J_{0}\left(k_{\perp} b_{\perp}\right) \\
& \times\left(\frac{\bar{x}}{x} F^{\prime 2}\left(z_{k}=\frac{\rho k_{\perp}}{2 \sqrt{x}}\right)+\frac{x}{\bar{x}} F^{\prime 2}\left(\bar{z}_{k}=\frac{\rho k_{\perp}}{2 \sqrt{\bar{x}}}\right)\right)
\end{aligned}
$$

where $F^{\prime}(z)$ is the $z$ derivative of Eq. (54).

In Fig. 15 we show the pion and kaon transverse spatial distributions from the QCD random instanton vacuum (87), at the low renormalization scale $Q_{0}=631 \mathrm{MeV}$. The corresponding distributions in transverse momentum space are also shown in Fig. 16 at the same scale.

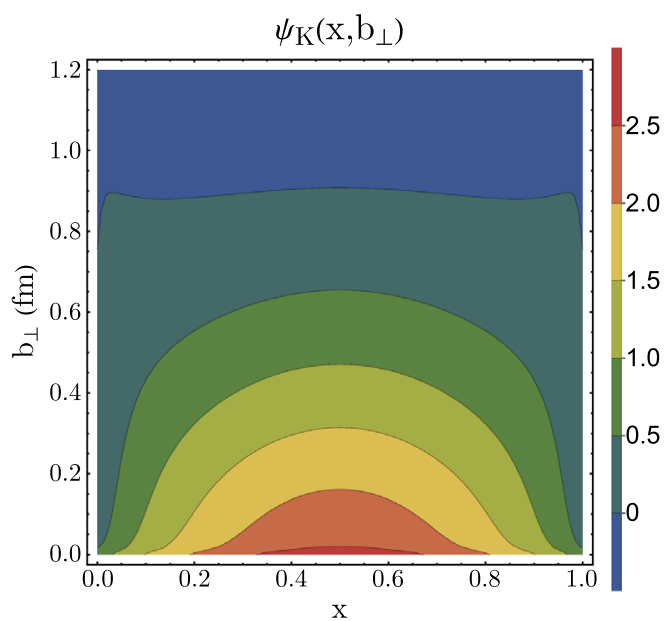

FIG. 15. Pion and kaon transverse spatial distribution from the QCD instanton vacuum (87) with physical masses and at the renormalization scale $Q_{0}=631 \mathrm{MeV}$. 

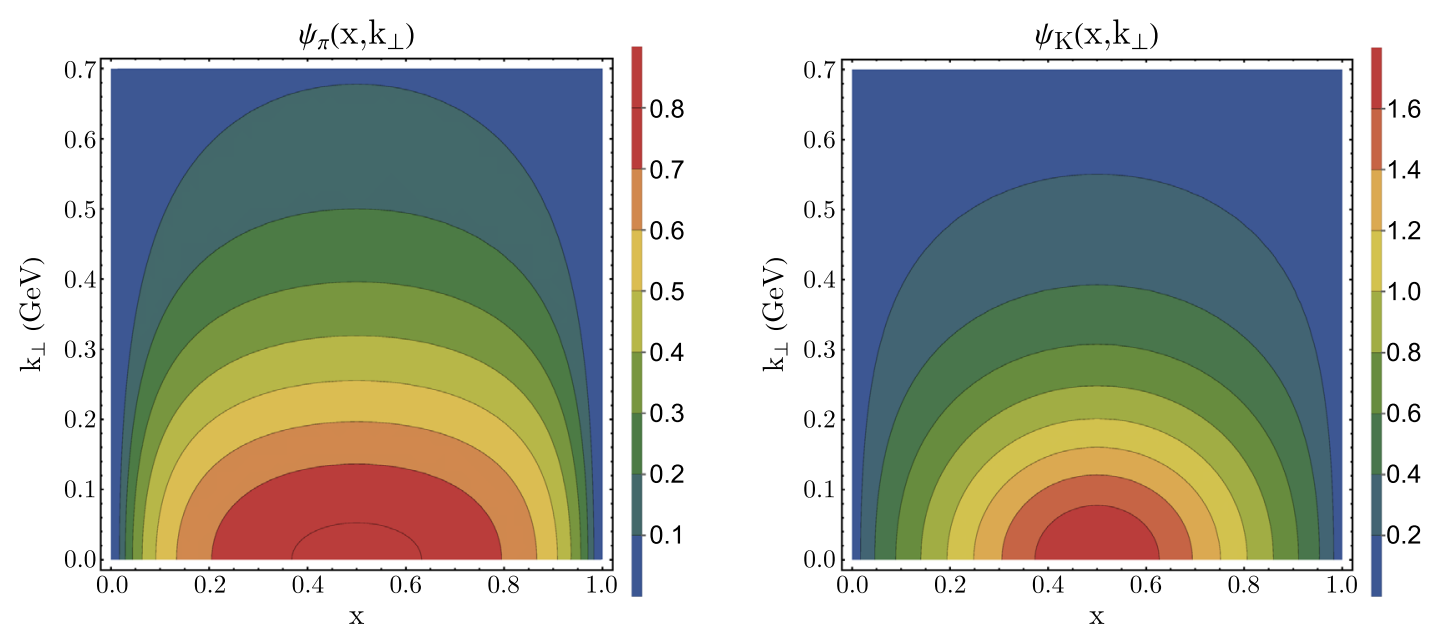

FIG. 16. Pion and kaon transverse momentum distribution from the QCD instanton vacuum (87) with physical masses and at the renormalization scale $Q_{0}=631 \mathrm{MeV}$.

\section{CONCLUSIONS}

We revisited the QCD instanton vacuum in the context of an exact planar resummation of the $n$-point correlations that preserves both gauge and chiral symmetry in power counting using the root of the packing fraction $\alpha \approx \sqrt{\kappa}$. We analyzed the induced quark mass, and effective pion pseudoscalar and pseudovector vertices at NLO with full conformity with the axial Ward identity in the chiral limit. Next-to-NLO contributions are readily available but tedious.

We used this framework to derive the soft contributions to the pion and kaon QPDA, QPDF and quasigeneralized distribution function (QGPDF) following from the QCD instanton vacuum. The results at LO show that these pion quasidistributions receive contributions from both the zero modes and the nonzero modes, but the latter contributions drop out in the large-momentum limit from the PDA and PDF. They are made explicit at LO or in the leadinglogarithm approximation.

The results we presented for the pion and kaon partonic distributions are all evaluated at the low renormalization scale set by the inverse instanton size $1 / \rho=631 \mathrm{MeV}$. A more compelling comparison with data at larger scales requires perturbative QCD evolution, assuming that factorization holds at this relatively low scale. Good agreement with the existing data for the pion PDA was found for all $x$, and the pion PDF at moderate $x$.

The present analysis of the pion and kaon quasiparton distributions relies on a diagrammatic expansion and power counting in $\alpha$ to analytically enforce chiral and gauge symmetry. It can be extended to all orders in $\alpha$ using welltested numerical simulations for the QCD instanton vacuum, that we will present elsewhere. In this respect, cooled lattice simulations of quasiparton distributions which are expected to be less noisy than the current simulations, would be welcome for comparison. The present results can be extended to the baryons away from the chiral limit.
One of the chief proposals for the forthcoming EIC is the understanding of the origin of mass and spin in most visible matter and its budgeting in terms of the fundamental constituents. The arguments we presented for the pion and kaon show that most of their composition is due to light quarks rescattering in a QCD instanton vacuum, giving rise to a large effective quark mass and small pion and kaon decay constants or chiral conductivity. The pion and kaon parton transverse distributions are controlled by the momentum-dependent effective quark mass, which is strongly sensitive to the instanton size but weakly sensitive to the current quark mass for the light $u, d, s$ quarks. The same mass and chiral conductivity control the valence partonic distributions of most light and heavy-light hadrons at low renormalization scale.

Finally, standard lore says that in light front quantization (LFQ) the vacuum is trivial. So how do we reconcile this with the present arguments that show that the quasiparton distributions for the light mesons carry vacuum physics all the way to the infinite-momentum limit? After all, the chiral condensate observed here is a scalar in all frames, including the light-cone frame. The answer lies in the neglected zero modes which when carefully treated in lower dimensions reproduce the chiral condensate in LFQ [33]. Recently, these zero modes were argued to accumulate at zero $x$ parton [34], much like a superfluid component in the otherwise normal fluid lightcone wave function, and show up as singular distributions in higher-twist observables as noted in Ref. [35]. It will be interesting to address these higher-twist distributions in the present context.

\section{ACKNOWLEDGMENTS}

We thank Edward Shuryak and Xiangdong Ji for discussions. This work was supported by the U.S. Department of Energy under Contract No. DE-FG-88ER40388. 


\section{APPENDIX A: ZERO MODES AND NONZERO- MODE QUARK PROPAGATOR}

In singular gauge, the instanton and anti-instanton quark zero modes in momentum space are locked in color-spin with a specific chirality

$$
\begin{aligned}
\psi_{0 I, \bar{I}}(p) & =\sqrt{2} \varphi^{\prime}(p) \hat{p} \chi^{ \pm} \\
\varphi^{\prime}(p) & =\pi \rho^{2}\left(I_{0}(z) K_{0}(z)-I_{1}(z) K_{1}(z)\right)_{z=p \rho / 2}^{\prime} .
\end{aligned}
$$

The prime denotes a $z$ derivative and $I, K$ are modified Bessel functions. The corresponding zero-mode projectors are

$P_{I}(p)=2 \varphi^{2} \hat{p} \chi^{+} \bar{\chi}^{-} \hat{p}=\frac{\varphi^{2}(p)}{8 p^{2}} \tau_{\mu}^{-} \tau_{\nu}^{+} \not p \gamma_{\mu} \gamma_{\nu} \not p \frac{1-\gamma_{5}}{2}$,

$P_{\bar{I}}(p)=2 \varphi^{\prime 2} \hat{p} \chi^{-} \bar{\chi}^{+} \hat{p}=\frac{\varphi^{\prime 2}(p)}{8 p^{2}} \tau_{\mu}^{+} \tau_{\nu}^{-} \not \gamma_{\mu} \gamma_{\nu} \not \not \frac{1+\gamma_{5}}{2}$

with $\tau_{\mu}^{ \pm}=(\vec{\tau}, \mp i)$. For comparison, note that the zero modes in regular gauge are simpler

$\psi_{0 I, \bar{I}}(p)=\sqrt{2} \varphi^{\prime}(p) \chi^{ \pm}, \quad \varphi^{\prime}(p)=4 \pi \rho^{2} e^{-p \rho} /(p \rho)$.

The nonzero mode are more involved to construct, but a closed form for their propagator is known in singular gauge [36]

$$
\begin{aligned}
G_{I}(x, y)= & \gamma_{\mu} D_{\mu}^{x} \Delta_{+}(x, y) \frac{1+\gamma_{5}}{2}+\Delta_{+}(x, y) \gamma_{\mu} D_{\mu}^{y} \frac{1-\gamma_{5}}{2}, \\
G_{\bar{I}}(x, y)= & \gamma_{\mu} D_{\mu}^{x} \Delta_{-}(x, y) \frac{1-\gamma_{5}}{2}+\Delta_{-}(x, y) \gamma_{\mu} D_{\mu}^{y} \frac{1+\gamma_{5}}{2} \\
\Delta_{ \pm}(x, y)= & \frac{1}{4 \pi^{2}(x-y)^{2}}\left(1+\frac{\rho^{2}}{x^{2}}\right)^{-\frac{1}{2}}\left(1+\frac{\rho^{2} \tau_{\mu}^{\mp} \tau_{\nu}^{ \pm} x_{\mu} y_{\nu}}{x^{2} y^{2}}\right) \\
& \times\left(1+\frac{\rho^{2}}{y^{2}}\right)^{-\frac{1}{2}}
\end{aligned}
$$

with the long derivative $D_{\mu}=\partial_{\mu}-i A_{\mu}$. Both at short and large distances Eq. (A4) reduces to the free propagator, while at intermediate distances it is modified. More specifically,

$G_{I}(x, y) \approx-\frac{1}{2 \pi^{2}} \frac{\gamma \cdot(x-y)}{(x-y)^{2}}-\frac{1}{16 \pi^{2}} \frac{(x-y)_{\mu} \gamma_{\nu} \gamma_{5}}{(x-y)^{2}} \tilde{F}_{\mu \nu}$

where $\tilde{F}$ is the dual of $F$. All omitted terms in Eq. (A5) are regular in the coincidental limit $x \rightarrow y$.

\section{APPENDIX B: PSEUDOVECTOR PION SOURCE AND AXIAL WARD IDENTITY}

In this appendix we detail the construction of the pseudovector pion source and show that it obeys a pertinent axial Ward identity at LO. The resummed planar approximation satisfies the strictures of gauge and chiral symmetry.

\section{Axial-vector pion vertex at LO}

For the pion axial correlator we insert

$$
O^{\mu 5}(P, k)=\gamma^{\mu} \gamma^{5}+\alpha F^{\mu 5}(P, k)+\mathcal{O}\left(\alpha^{2}\right)
$$

into Eq. (19), and use the LO contribution for the quark propagator in Eq. (8) and the NLO contribution for the spin-valued self-energy (16). Power matching in $\alpha$ yields the spin-valued integral equation

$$
\begin{aligned}
F^{\mu 5}(P, k)= & K_{\pi} F^{\mu 5}(P, k) \\
& +\sum_{I, \bar{I}} \int \frac{d^{4} p}{(2 \pi)^{4}}\left(\operatorname{Tr}_{C} \Sigma_{I 0}(k, p) \frac{i \sigma}{p^{2}} \gamma^{\mu} \gamma^{5} S_{0}\left(p_{-}\right) \Sigma_{I 0}\left(p_{-}, k_{-}\right)+\operatorname{Tr}_{C} \Sigma_{I 0}(k, p) S_{0}(p) \gamma^{\mu} \gamma^{5} \frac{i \sigma}{\left(p_{-}\right)^{2}} \Sigma_{I 0}\left(p_{-}, k_{-}\right)\right. \\
& \left.+\operatorname{Tr}_{C} \Sigma_{I 1}(k, p) S_{0}(p) \gamma^{\mu} \gamma^{5} S_{0}\left(p_{-}\right) \Sigma_{I 0}\left(p_{-}, k_{-}\right)+\operatorname{Tr}_{C} \Sigma_{I 0}(k, p) S_{0}(p) \gamma^{\mu} \gamma^{5} S_{0}\left(p_{-}\right) \Sigma_{I 1}\left(p_{-}, k_{-}\right)\right) .
\end{aligned}
$$

Here $p_{-}=p-P$. The reduced kernel $K_{\pi}$ involves only the zero modes and satisfies

$$
K_{\pi} O=\sum_{I, \bar{I}} \int \frac{d^{4} p}{(2 \pi)^{4}} \operatorname{Tr}_{C}\left(\Sigma_{I 0}(k, p) S_{0}(p) O S_{0}\left(p_{-}\right) \Sigma_{I 0}\left(p_{-}, k_{-}\right)\right) .
$$

The $\beta_{00}$ contribution in $\Sigma_{I 1}$ in Eq. (16) does not contribute to this order, and the $\left(i \not \partial P_{I} \hat{\sigma}+i \hat{\sigma} P_{I} \not \partial\right)$ contribution cancels exactly the first two terms in Eq. (B2). The final relation for $F^{\mu 5}$ simplifies 


$$
\begin{aligned}
F^{\mu 5}(P, k)= & K_{\pi} F^{\mu 5}(P, k)+\sum_{I+\bar{I}} \int \frac{d^{4} p}{(2 \pi)^{4}}\left(\operatorname{Tr}_{C}\left(\not k \tilde{G}_{I}(k, p) \gamma^{\mu} \gamma^{5} \psi_{0}\left(p_{-}\right) \psi_{0}^{\dagger}\left(k_{-}\right) \frac{\not k_{-}}{i \sigma_{00}}\right)\right. \\
& \left.+\operatorname{Tr}_{C}\left(\frac{\not k}{i \sigma_{00}} \psi_{0}(k) \psi_{0}^{\dagger}(p) \gamma^{\mu} \gamma^{5} \tilde{G}^{I}\left(p_{-}, k_{-}\right) k_{-}\right)\right) .
\end{aligned}
$$

Here

$$
\tilde{G}_{I}=\left(1-P_{I} \hat{\sigma}\right) G_{I}\left(1-\hat{\sigma} P_{I}\right)-S_{0}
$$

is the projected and subtracted nonzero-mode propagator which is UV finite. The only nonvanishing contributions to Eq. (B4) are

$$
-P_{I} \hat{\sigma} G_{I}-G_{I} \hat{\sigma} P_{I}+G_{I}-S_{0} .
$$

If we approximate $G_{I} \approx S_{0}$, then Eq. (B4) will reduce to the first two contributions in Eq. (B2) only. This corresponds to expanding the propagator to first order while keeping all $\Sigma$ 's unchanged. However, this approximation upsets the axial Ward identity.

\section{Axial Ward identity at LO}

In the chiral limit the pseudovector pion vertex satisfies the exact Ward identity

$$
P_{\mu} O^{\mu 5}(k, P)=\gamma^{5} S^{-1}(k-P)+S^{-1}(k) \gamma^{5}
$$

to all orders in $\alpha$, which guarantees the transversality of the axial-vector correlator

$$
\begin{aligned}
P_{\nu} & \left\langle F^{\mu 5}(-P) F^{\nu 5}(P)\right\rangle \\
& =\int \frac{d^{4} k}{(2 \pi)^{4}} \operatorname{Tr}_{C}\left(\gamma^{\mu} \gamma^{5}\left(S^{-1}(k) \gamma^{5}+\gamma^{5} S^{-1}(k-P)\right)\right)=0
\end{aligned}
$$

since $S^{-1}(k)=\not k-i \sigma(k)$. The enforcement of the Ward identity and power counting guarantees chiral and gauge symmetry. In particular, the extraction of the pion decay constant in power counting whether from the pseudoscalar vertex or the pseudovector vertex is unique order by order in $\alpha$. This is not the case in the partial resummations used in Refs. [6,7] where different values of $f_{\pi}$ were noted. Since the normalization of the PDA and PDF involve $f_{\pi}$, the strict enforcement of the Ward identities is required.

Equation (B7) uniquely fixes the longitudinal part of the pseudovector pion vertex to all orders in $\alpha$

$$
F_{L}^{\mu 5}(k, P)=-i \gamma_{5}(\sigma(k)+\sigma(k-P)) \frac{P_{\mu}}{P^{2}} .
$$

In contrast, the transverse part is more involved, and can only be obtained through an expansion. At LO

$$
\begin{aligned}
F^{\mu 5}(k, P)= & \lambda(P)\left\langle 0\left|\delta F^{\mu 5}(k, P)\right| 0\right\rangle \gamma_{5}|k||k-P| \varphi^{\prime}(k) \varphi^{\prime}(k-P) \\
& +\delta F^{\mu 5}(k, P) .
\end{aligned}
$$

Here $\delta F^{\mu 5}$ refers to the inhomogeneous contribution in Eq. (B4) to order $\alpha$. The pion pole resides in $\lambda(P)$ with $\delta F^{\mu 5}$ regular at $P^{2}=0$. Since $\left\langle 0\left|\delta F^{\mu 5}\right| 0\right\rangle$ is of the form $\sim k^{\mu} \gamma^{5}$ or $P^{\mu} \gamma^{5}$, it follows that the axial-axial vector correlation function vanishes to order $\alpha$. We expect the axial-axial vector correlator to be transverse and of order $f_{\pi}^{2} \sim \alpha^{2}$, as we now show.

We now proceed to show that our power counting enforces Eq. (B8) order by order. For that, consider the contribution $-P^{I} \hat{\sigma} G^{I}-G^{I} \hat{\sigma} P^{I}$ in the inhomogeneous part of Eq. (B4), and contract it with $P_{\mu}$. The result is

$$
\begin{array}{r}
-\operatorname{Tr}_{C} \not \not \psi_{I 0}(p) A_{I}^{\mu}(P) \psi_{I 0}^{\dagger}(p-P) \frac{\not p-\not p}{i \sigma_{00}} \\
-\operatorname{Tr}_{C} \not \not \psi_{I 0}(p) B_{I}^{\mu}(P) \psi_{I 0}^{\dagger}(p-P) \frac{\not p-\not P}{i \sigma_{00}}
\end{array}
$$

where we have defined

$$
\begin{aligned}
A_{I}^{\mu} & =\int \frac{d^{4} k}{(2 \pi)^{4}} \beta^{\dagger}(p) \gamma^{\mu} \gamma^{5} \psi_{I 0}(k-P), \\
B_{I}^{\mu} & =\int \frac{d^{4} k}{(2 \pi)^{4}} \psi_{I 0}^{\dagger}(p) \gamma^{\mu} \gamma^{5} \beta(p-P)
\end{aligned}
$$

with

$$
\beta(p)=\int \frac{d^{4} k}{(2 \pi)^{4}} G_{I}(p, k) \hat{\sigma}(k) \psi_{I 0}(k)
$$

or equivalently ( $x$ space)

$$
\begin{aligned}
& A_{I}=\int d^{4} x \beta^{\dagger}(x) \gamma^{\mu} \gamma_{5} \psi_{I 0}(x) e^{i P x}, \\
& B_{I}=\int d^{4} x \psi_{I 0}^{\dagger}(x) \gamma^{\mu} \gamma_{5} \beta(x) e^{i P x}
\end{aligned}
$$

so that 


$$
\begin{aligned}
& P_{\mu} B_{I}^{\mu}=\int d^{4} x\left(-i \not_{I} \psi_{0 I}\right)^{\dagger} \gamma_{5} \beta(x) e^{i P x}-\int d^{4} x \psi_{0 I}^{\dagger} \gamma_{5} i \not_{I} \beta(x) e^{i P x}, \\
& P_{\mu} A_{I}^{\mu}=\int d^{4} x\left(-i \not D_{I} \beta\right)^{\dagger} \gamma_{5} \psi_{0 I}(x) e^{i P x}-\int d^{4} x \beta^{\dagger} \gamma_{5} i \not_{I} \psi_{0 I}(x) e^{i P x} .
\end{aligned}
$$

From Eq. (B13) it follows that $\beta(x)=\int d^{4} d d^{4} y G^{I}(x, z) \hat{\sigma}(z-y) \psi_{0}(y)$, so that the action of $\not_{I}$ on $\beta(x)$ is fixed by the zero mode only, and similarly for the contribution with $G^{I}$, which gives

$$
\operatorname{Tr}_{C} \not \not C_{I}^{\mu} \psi_{0 I}^{\dagger}(p-P) \frac{\not p-\not p}{i \sigma_{00}}+\operatorname{Tr}_{C} \frac{\not p}{i \sigma_{00}} \psi_{0 I}(p) D_{I}^{\mu}(\not p-\not p)
$$

with

$$
\begin{aligned}
& C_{I}^{\mu}=\int d^{4} y G^{I}(p, y) \gamma^{\mu} \gamma^{5} \psi_{0 I}(y) e^{i P y}, \\
& D_{I}^{\mu}=\int d^{4} y \psi_{0 I}^{\dagger} \gamma^{\mu} \gamma^{5} G^{I}(y, p-P) e^{i P y} .
\end{aligned}
$$

They can be simplified using the same observations. Hence, after contracting with $P_{\mu}$ the results are

$$
\begin{aligned}
P_{\mu}\left(A_{I}^{\mu}+B_{I}^{\mu}\right) & =\int \frac{d^{4} p}{(2 \pi)^{4}} \psi_{0 I}^{\dagger}(p) \gamma^{5} \psi_{0 I}(p-P)\left(2-\frac{\sigma_{p}+\sigma_{p-P}}{\sigma_{00}}\right), \\
P_{\mu} C_{I}^{\mu} & =-\gamma_{5} \psi_{0 I}(p-P)+\psi_{0 I}(p) \int \frac{d^{4} k}{(2 \pi)^{4}} \psi_{0 I}^{\dagger}(k) \gamma^{5} \psi_{0 I}(k-P), \\
P_{\mu} D_{I}^{\mu} & =-\psi_{0 I}^{\dagger}(p) \gamma_{5}+\int \frac{d^{4} k}{(2 \pi)^{4}} \psi_{0 I}^{\dagger}(k) \gamma^{5} \psi_{0 I}(k-P) \psi_{0 I}^{\dagger}(p-P) .
\end{aligned}
$$

Finally, the contribution with $1 / i \not \partial$ can be directly calculated and gives after contracting with $P_{\mu}$

$$
\operatorname{Tr}_{C} \not P \gamma^{5} \psi_{0 I}(p-P) \psi_{0 I}^{\dagger}(p-P) \frac{\not p-\not p}{i \sigma_{00}}-\operatorname{Tr}_{C} \frac{\not p}{i \sigma_{0} 0} \psi_{0}(p) \psi_{0 I}^{\dagger}(p) \gamma^{5} \not p .
$$

While combining the above results, we note that the second terms in $C$ and $D$ cancel with the 2 in the parentheses $\left(2-\sigma / \sigma_{00}\right)$ for $A$ and $B$, and the contributions $\gamma^{5} \psi_{0}$ and $\psi_{0}^{\dagger} \gamma^{5}$ in $C$ and $D$ respectively, combine with the contribution $1 / i \not \partial$ to give $\not p-\not p$ or $\not p$ respectively. The final result after contracting with $P_{\mu}$ is

$$
\begin{aligned}
& \sum_{I} \operatorname{Tr}_{C}\left(\not p \psi_{0 I}(p)\left(\int \frac{d^{4} k}{(2 \pi)^{4}} \psi_{0 I}^{\dagger}(k) \frac{\sigma_{k}+\sigma_{k-P}}{\sigma_{00}} \gamma_{5} \psi_{0 I}(k-P)\right) \psi_{0 I}^{\dagger}(p-P) \frac{\not p-\not p}{i \sigma_{00}}\right) \\
& +\sum_{I} \operatorname{Tr}_{I}\left(\gamma_{5}(p-\not p) \frac{\psi_{0 I}(p-P) \psi_{0 I}^{\dagger}(p-P)}{i \sigma_{00}}(\not p-\not p)\right)+\sum_{I} \operatorname{Tr}_{C}\left(\gamma_{5} \not \not p \frac{\psi_{0 I}(p) \psi_{0 I}^{\dagger}(p)}{i \sigma_{00}} \not p\right) .
\end{aligned}
$$

Using the definition of $K_{\pi}$ in Eq. (B3) and the gap equation for $\sigma$, Eq. (B20) can be written as

$$
\left(1-K_{\pi}\right)\left(-i \gamma^{5} \sigma(k)-i \gamma^{5} \sigma(k-P)\right)
$$

which is the action of $P_{\mu}$ on the inhomogeneous part of Eq. (B4), or

$$
\left(1-K_{\pi}\right)\left(P_{\mu} F^{\mu 5}(k, P)\right)=\left(1-K_{\pi}\right)\left(-i \gamma^{5} \sigma(k)-i \gamma^{5} \sigma(k-P)\right) .
$$

It follows that

$$
P_{\mu} F^{\mu 5}(k, P)=-i \gamma^{5} \sigma(k)-i \gamma^{5} \sigma(k-P)
$$


which is the axial Ward identity expanded to first order in $\alpha$. This concludes our proof that Eq. (B1) and the corresponding two-point correlation function satisfy the axial Ward identity at LO in $\alpha$.

\section{APPENDIX C: GAUGE LINK}

We can show that to the same order $\alpha^{0}$ the only contribution of the gauge link in Eq. (39) follows from Eq. (45) with the substitution

$$
\int \frac{d^{3} p}{(2 \pi)^{3}} \psi_{0 I}^{\dagger}\left(p^{-}\right) \gamma^{z} \gamma^{5} \delta G_{I}(p, k) \rightarrow \int d^{4} y d z e^{-i P y+i P_{z}\left(x-\frac{1}{2}\right)} z \psi_{0 I}^{\dagger}\left(y+\frac{z}{2}\right) \gamma^{z} \gamma^{5}\left[y+\frac{z}{2}, y-\frac{z}{2}\right]_{I} \delta G_{I}\left(y-\frac{z}{2}, k\right)
$$

for the first term, and similarly for the second term. The gauge link involves the $z$ propagation of a quark in a single instanton,

$$
\left[y+\frac{z}{2}, y-\frac{z}{2}\right]_{I}=\left\langle y+\frac{z}{2}\left|\frac{1}{i \partial_{z}-A_{I z}}\right| y-\frac{z}{2}\right\rangle
$$

and restores explicit gauge invariance in Eq. (45) to order $\alpha^{0}$. For instance, in the regular gauge with $A_{M}=-\bar{\sigma}_{M N} x_{N} \frac{1}{x^{2}+\rho^{2}}$, the gauge link simplifies

$$
\left[y+\frac{z}{2}, y-\frac{z}{2}\right]_{I, \bar{I}}=\cos F\left(r_{3}, y_{z}, z\right) \pm i \sigma \cdot \hat{r}_{3} \sin F\left(r_{3}, y_{z}, z\right)
$$

with

$$
F\left(r_{3}, y_{z}, z\right)=\int_{-1}^{1} d \tau\left[\frac{r_{3} \frac{z}{2}}{r_{3}^{2}+\rho^{2}+\left(y_{z}+\frac{\tau z}{2}\right)^{2}}\right]=\frac{r_{3}}{\sqrt{r_{3}^{2}+\rho^{2}}}\left[\arctan \left(\frac{y_{z}+\frac{z}{2}}{\left(r_{3}^{2}+\rho^{2}\right)^{\frac{1}{2}}}\right)-\arctan \left(\frac{y_{z}-\frac{z}{2}}{\left(r_{3}^{2}+\rho^{2}\right)^{\frac{1}{2}}}\right)\right]
$$

\section{APPENDIX D: GENERALIZED QPDA}

The pion QPDA (18) in the random instanton vacuum is part of a larger class of quasidistributions. For instance, the LO contribution (47) can be recast in the general form

$$
\tilde{\phi}_{\pi}^{0}(x, n, P)=-\frac{4 i N_{c}}{f_{\pi}^{2}} \int \frac{d^{4} k}{(2 \pi)^{4}} \delta\left(n \cdot k-\left(x-\frac{1}{2}\right) n \cdot P\right)\left(M\left(y_{1}\right) M\left(y_{2}\right)\right)^{\frac{1}{2}} \frac{\left(n \cdot p_{1} M\left(y_{2}\right)+n \cdot p_{2} M\left(y_{1}\right)\right)}{y_{1}^{2} y_{2}^{2}}
$$

where $n$ is an arbitrary 4-vector, using Minkowski signature and the causal assignment for the poles. Lorentz and "scale" invariance imply

$$
\tilde{\phi}_{\pi}^{0}(x, n, P) \equiv \phi_{\pi}\left(x, P^{2}, \frac{n^{2}}{(n \cdot P)^{2}}\right)
$$

For time-like $n=n_{-}$and space-like $n=n_{z}$ we have respectively the PDA and QPDA, i.e.,

$\phi_{\pi}^{0}(x)=\tilde{\phi}_{\pi}^{0}\left(x, m_{\pi}^{2}, 0\right), \quad \tilde{\phi}_{\pi}^{0}\left(x, P_{z}\right)=\tilde{\phi}_{\pi}^{0}\left(x, m_{\pi}^{2}, \frac{1}{P_{z}^{2}}\right)$
For large $P_{z}$, the pion QPDA reduces to the PDA in the random instanton vacuum.

\section{APPENDIX E: ZERO AND NONZERO MODES ON SHELL}

The zero and nonzero modes entering our analysis of the PDA and PDF simplify when they are put on mass shell which is the leading contribution for the quasidistributions in the large- $P_{z}$ limit. For the zero modes, the mass-shell reduction yields constant Weyl spinors. From Eq. (A1) we have for the zero modes

$$
\not \supset \psi_{0 I, 0 \bar{I}}(p) \rightarrow-\sqrt{2} \pi \rho \chi^{ \pm}
$$


with $p^{2}=0$. For the nonzero modes we have

$$
\begin{aligned}
& \int d^{4} x e^{-i q \cdot x} \psi_{0 I}^{+}(x) \bar{\sigma}_{z} \delta G_{I}(x, p) i \bar{p} \rightarrow \mathbb{F}(q, p), \\
& \int d^{4} x e^{-i q \cdot x} i p \delta \bar{G}_{I}(p, x) \sigma_{z} \psi_{0 I}(x) \rightarrow \overline{\mathbb{F}}(q, p)
\end{aligned}
$$

with $\delta \bar{G}_{I}$ following from $\delta G_{I}$ by barring the Weyl contributions. A tedious derivation following the arguments presented in Ref. [37] gives for the mixed and subtracted instanton nonzero-mode contributions the results in Eq. (60).

\section{APPENDIX F: PION QGPDF AT LO}

The pion QGPDF can also be extracted from the equaltime correlator following Eq. (1) as suggested in Ref. [3], with formally

$$
\tilde{\Psi}_{\pi}(x, q, P)=\int \frac{d z}{2 \pi} e^{-i(x-\bar{x}) z p_{z}}\left\langle\pi(P+q)\left|\bar{\Psi}\left(z_{-}\right) \gamma^{z}\left[z_{-}, z_{+}\right] \Psi\left(z_{+}\right)\right| \pi(P)\right\rangle
$$

In the random instanton vacuum Eq. (F1) follows from the same reduction rules as those for the quasi-distribution amplitude (QDA) and QPDA detailed above. Both the zero modes and nonzero modes contribute to order $\alpha^{0}$ to LO, but the dominant contribution stems from the zero modes in the large-momentum limit as we noted earlier. The LO result for the QGPDF after spin-color contractions and the free approximation for the nonzero modes $\delta G_{I} \approx S_{0}$, is

$$
\tilde{\Psi}_{\pi}^{0}(x, q, P) \approx \frac{4 i N_{c}}{f_{\pi}^{2}} \int \frac{d k_{4} d^{2} k_{\perp}}{(2 \pi)^{4}}\left(M\left(k_{1}\right) M^{2}\left(k_{2}\right) M\left(k_{3}\right)\right)^{\frac{1}{2}}\left(\frac{k_{1}^{z}+k_{3}^{z}}{2 k_{1}^{2} k_{3}^{2}}+\frac{k_{1}^{z}}{2 k_{1}^{2} k_{2}^{2}}+\frac{k_{3}^{z}}{2 k_{2}^{2} k_{3}^{2}}-\frac{k_{2}^{z}}{k_{2}^{2} k_{3}^{2}}-\frac{k_{2}^{z} k_{1} \cdot q}{k_{1}^{2} k_{2}^{2} k_{3}^{2}}\right)+\operatorname{cross}
$$

with $k_{\perp} \geq 0$ subsumed, $k_{z}=x P_{z}$ and

$$
k_{1}=k-\frac{q}{2}, \quad k_{2}=-P-\frac{q}{2}+k, \quad k_{3}=k+\frac{q}{2} .
$$

The cross terms have the same structure but with the substitution $k_{2} \rightarrow k_{2}^{\prime}=P+k+\frac{q}{2}$. The GPDF follows from Eq. (F2) in the large- $P_{z}$ limit. It will be analyzed elsewhere.

[1] G. R. Farrar and D. R. Jackson, Phys. Rev. Lett. 43, 246 (1979).

[2] J. H. Zhang, J. W. Chen, X. Ji, L. Jin, and H. W. Lin, Phys. Rev. D 95, 094514 (2017).

[3] X. Ji, Phys. Rev. Lett. 110, 262002 (2013).

[4] M. C. Chu, J. M. Grandy, S. Huang, and J. W. Negele, Phys. Rev. D 49, 6039 (1994).

[5] E. V. Shuryak, Nucl. Phys. B319, 541 (1989); T. Schafer and E. V. Shuryak, Rev. Mod. Phys. 70, 323 (1998).

[6] D. Diakonov and V. Y. Petrov, Nucl. Phys. B272, 457 (1986); D. Diakonov, Prog. Part. Nucl. Phys. 51, 173 (2003).

[7] M. A. Nowak, J. J. M. Verbaarschot, and I. Zahed, Nucl. Phys. B325, 581 (1989); M. Kacir, M. Prakash, and I. Zahed, Acta Phys. Pol. B 30, 287 (1999); M. A. Nowak, M. Rho, and I. Zahed, Chiral Nuclear Dynamics (World Scientific, Singapore, 1996), p. 528.

[8] A. V. Radyushkin, in Proceedings, Continuous advances in $Q C D$, Minneapolis, Minnesota, edited by A.V. Smilga (World Scientific, River Edge, 1994), pp. 238-248.

[9] S. J. Brodsky, F. G. Cao, and G. F. de Teramond, Phys. Rev. D 84, 033001 (2011); S. J. Brodsky, G. F. de Teramond, H. G. Dosch, and J. Erlich, Phys. Rep. 584, 1 (2015).
[10] L. Chang, I. C. Cloet, J. J. Cobos-Martinez, C. D. Roberts, S. M. Schmidt, and P.C. Tandy, Phys. Rev. Lett. 110, 132001 (2013); C. Chen, L. Chang, C. D. Roberts, S. Wan, and H. S. Zong, Phys. Rev. D 93, 074021 (2016); M. Ding, K. Raya, D. Binosi, L. Chang, C. D. Roberts, and S. M. Schmidt, Phys. Rev. D 101, 054014 (2020).

[11] S. Jia and J. P. Vary, Phys. Rev. C 99, 035206 (2019); J. Lan, C. Mondal, S. Jia, X. Zhao, and J. P. Vary, Phys. Rev. Lett. 122, 172001 (2019); E. Shuryak, Phys. Rev. D 100, 114018 (2019).

[12] W. Broniowski and E. Ruiz Arriola, Phys. Lett. B 773, 385 (2017); Proc. Sci., Hadron2017 (2018) 174 [arXiv:1711 .09355]; Phys. Rev. D 97, 034031 (2018); Phys. Lett. B 773, 385 (2017); A. E. Dorokhov, W. Broniowski, and E. Ruiz Arriola, Phys. Rev. D 84, 074015 (2011); W. Broniowski, E. Ruiz Arriola, and K. Golec-Biernat, Phys. Rev. D 77, 034023 (2008); E. Ruiz Arriola and W. Broniowski, Phys. Rev. D 66, 094016 (2002); M. Praszalowicz and A. Rostworowski, Phys. Rev. D 97, 034031 (2018); M. Praszalowicz and A. Rostworowski, Acta Phys. Pol. B 34, 2699 (2003); D. G. Dumm, S. Noguera, N. N. Scoccola, and S. Scopetta, Phys. Rev. D 89, 054031 (2014). 
[13] V. Y. Petrov and P. V. Pobylitsa, arXiv:hep-ph/9712203; V. Y. Petrov, M. V. Polyakov, R. Ruskov, C. Weiss, and K. Goeke, Phys. Rev. D 59, 114018 (1999).

[14] I. V. Anikin, A. E. Dorokhov, and L. Tomio, Yad. Fiz. 64, 1405 (2001) [Phys. At. Nucl. 64, 1329 (2001)]; A. E. Dorokhov and L. Tomio, Phys. Rev. D 62, 014016 (2000); S. i. Nam, H. C. Kim, A. Hosaka, and M. M. Musakhanov, Phys. Rev. D 74, 014019 (2006); S. i. Nam and H. C. Kim, Phys. Rev. D 74, 076005 (2006); A. E. Dorokhov, Braz. J. Phys. 37, 819 (2007) [Czech. J. Phys. 56, F169 (2006)]; A. E. Dorokhov, Pis'ma Zh. Eksp. Teor. Fiz. 77, 68 (2003) [JETP Lett. 77, 63 (2003)]; A. E. Dorokhov and L. Tomio, arXiv:hep-ph/9803329; A. E. Dorokhov, Nuovo Cimento A 109, 391 (1996).

[15] X. Ji, A. Schafer, X. Xiong, and J. H. Zhang, Phys. Rev. D 92, 014039 (2015).

[16] J. H. Zhang, J. W. Chen, X. Ji, L. Jin, and H. W. Lin, Phys. Rev. D 95, 094514 (2017); G. S. Bali et al., Phys. Rev. D 98, 094507 (2018); C. Alexandrou, K. Cichy, M. Constantinou, K. Jansen, A. Scapellato, and F. Steffens, Phys. Rev. D 98, 091503 (2018); T. Ishikawa, L. Jin, H. W. Lin, A. Schafer, Y. B. Yang, J. H. Zhang, and Y. Zhao, Sci. China Phys. Mech. Astron. 62, 991021 (2019); T. Izubuchi, L. Jin, C. Kallidonis, N. Karthik, S. Mukherjee, P. Petreczky, C. Shugert, and S. Syritsyn, Phys. Rev. D 100, 034516 (2019).

[17] A. V. Radyushkin, Phys. Rev. D 95, 056020 (2017).

[18] S. i. Nam, Mod. Phys. Lett. A 32, 1750218 (2017); W. Broniowski and E. Ruiz Arriola, Phys. Lett. B 773, 385 (2017).

[19] P. V. Pobylitsa, Phys. Lett. B 226, 387 (1989).

[20] N. Andrei and D. J. Gross, Phys. Rev. D 18, 468 (1978).
[21] C. Michael and P. S. Spencer, Phys. Rev. D 52, 4691 (1995).

[22] P. O. Bowman, U. M. Heller, D. B. Leinweber, A. G. Williams, and J.b. Zhang, Nucl. Phys. B, Proc. Suppl. 128, 23 (2004).

[23] H. D. Politzer, Nucl. Phys. B117, 397 (1976).

[24] R. A. Janik, M. A. Nowak, G. Papp, and I. Zahed, Phys. Rev. Lett. 81, 264 (1998).

[25] G. P. Lepage and S. J. Brodsky, Phys. Lett. 87B, 359 (1979); A. V. Efremov and A. V. Radyushkin, Phys. Lett. 94B, 245 (1980).

[26] D. Diakonov, V. Y. Petrov, and P. V. Pobylitsa, Phys. Lett. B 226, 372 (1989); S. Chernyshev, M. A. Nowak, and I. Zahed, Phys. Lett. B 350, 238 (1995).

[27] X. Ji, Y. Liu, and I. Zahed, Phys. Rev. D 99, 054008 (2019).

[28] E. M. Aitala et al. (E791 Collaboration), Phys. Rev. Lett. 86, 4768 (2001).

[29] J. S. Conway et al., Phys. Rev. D 39, 92 (1989).

[30] M. Aicher, A. Schafer, and W. Vogelsang, Phys. Rev. Lett. 105, 252003 (2010).

[31] A. C. Aguilar et al., Eur. Phys. J. A 55, 190 (2019).

[32] R. S. Sufian et al., arXiv:2001.04960.

[33] F. Lenz, M. Thies, K. Yazaki, and S. Levit, Ann. Phys. (N.Y.) 208, 1 (1991); K. Hornbostel, Phys. Rev. D 45, 3781 (1992); C. R. Ji and S. J. Rey, Phys. Rev. D 53, 5815 (1996).

[34] X. Ji, arXiv:2003.04478.

[35] F. Aslan and M. Burkardt, Phys. Rev. D 101, 016010 (2020).

[36] L. S. Brown, R. D. Carlitz, D. B. Creamer, and C. k. Lee, Phys. Rev. D 17, 1583 (1978).

[37] S. Moch, A. Ringwald, and F. Schrempp, Nucl. Phys. B507, 134 (1997). 